•综述・

\title{
遥感在生物多样性研究中的应用进展
}

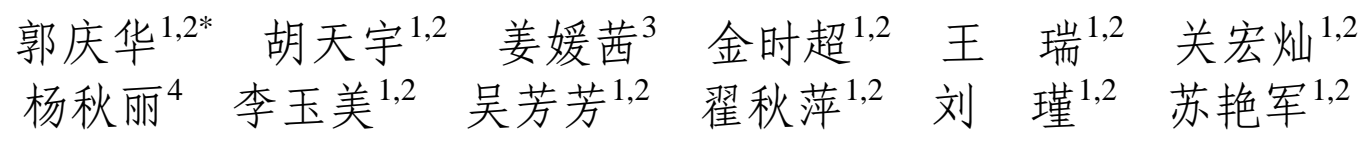

1 (中国科学院植物研究所植被与环境变化国家重点实验室, 北京 100093)

2 (中国科学院大学, 北京 100049)

3 (北京城市学院城市建设学部, 北京 100083)

4 (新疆大学资源与环境科学学院, 乌鲁木齐 830046)

\begin{abstract}
摘要: 随着人口的持续增长, 人类经济活动对自然资源的利用强度不断升级以及全球气候变暖, 全球物种正以前 所未有的速度丧失, 生物多样性成为了全球关注的热点问题。传统生物多样性研究以地面调查方法为主, 重点关 注物种或样地水平, 但无法满足景观尺度、区域尺度以及全球尺度的生物多样性保护和评估需求。遥感作为获取 生物多样性信息的另一种手段, 近年来在生物多样性领域发展迅速, 其覆盖广、序列性以及可重复性等特点使之 在大尺度生物多样性监测和制图以及评估方面具有极大优势。本文主要通过文献收集整理, 从观测手段、研究尺 度、观测对象和生物多样性关注点等方面综述了遥感在生物多样性研究中的应用现状, 重点分析不同遥感平台的 技术优势和局限性, 并探讨了未来遥感在生物多样性研究的应用趋势。遥感平台按观测高度可分为近地面遥感、 航空遥感和卫星遥感, 能够获取样地-景观-区域-洲际-全球尺度的生物多样性信息。星载平台在生物多样性研究 中应用最多, 航空遥感的应用研究偏少主要受飞行成本限制。近地面遥感作为一个新兴平台, 能够直接观测到物 种的个体, 获取生物多样性关注的物种和种群信息, 是未来遥感在生物多样性应用中的发展方向。虽然遥感技术 在生物多样性研究中的应用存在一定的局限性, 未来随着传感器发展和多源数据融合技术的完善, 遥感能更好地 从多个尺度、全方位地服务于生物多样性保护和评估。
\end{abstract}

关键词: 卫星遥感; 航空遥感; 近地面遥感; 无人机; 激光雷达

\section{Advances in remote sensing application for biodiversity research}

Qinghua Guo ${ }^{1,2^{*}}$, Tianyu $\mathrm{Hu}^{1,2}$, Yuanxi Jiang ${ }^{3}$, Shichao $\mathrm{Jin}^{1,2}$, Rui Wang ${ }^{1}$, Hongcan Guan ${ }^{1,2}$, Qiuli Yang ${ }^{4}$, Yumei $\mathrm{Li}^{1,2}$, Fangfang $\mathrm{Wu}^{1,2}$, Qiuping Zhai ${ }^{1,2}$, Jin Liu ${ }^{1,2}$, Yanjun $\mathrm{Su}^{1,2}$

1 State Key Laboratory of Vegetation and Environmental Change, Institute of Botany, Chinese Academy of Sciences, Beijing 100093

2 University of Chinese Academy of Sciences, Beijing 100049

3 Urban Construction School, Beijing City University, Beijing 100083

4 College of Resource and Environment Sciences, Xinjiang University, Urumqi 830046

\begin{abstract}
Since rapid human population growth, overconsumption of natural resources by human activities and climate change, loss and extinction of species is increasing, and biodiversity become an important global issue. Traditional ground-based biodiversity researches focus on the species or community, which can not provide necessary information for biodiversity conservation and assessment at a large scale. Since the advantages in spatial coverage and time series, remote sensing is very useful in large-scale biodiversity monitoring, mapping and assessment. According to the height of the platform, remote sensing platforms can be classified into satellite remote sensing, airborne remote sensing and near-surface remote sensing, which can obtain biodiversity information at different spatial scales. The purpose of this study is to review the recent advances of application of different remote sensing platforms for biodiversity research. We focus on the following aspects, such as observation methods, research scale, and analyze advantages and limitations of different remote
\end{abstract}

收稿日期: 2018-07-22; 接受日期: 2018-07-24

基金项目: 国家重点研发计划(2016YFC0500202)和 2017 年北京高等学校高水平人才交叉培养“实培计划”项目

* 通讯作者 Author for correspondence. E-mail: qguo@ibcas.ac.cn 
sensing platforms. Finally, we summary the future application of remote sensing in biodiversity research. From the literature statistics result, we found that satellite platform were used more frequently in biodiversity research than other remote sensing platform. Due to the high flight cost, the biodiversity researches used airborne remote sensing was fewer than the researches used satellite. Near-surface remote sensing includes the UAV platform and the ground-based platform, which is an emerging remote sensing platform and hotspot in remote sensing of biodiversity. Compared to satellite and airborne remote sensing platforms, the near-surface remote sensing platform can directly observe the individuals and can directly obtain information from species or population. Although there are some limitations in these three platforms, we believe that remote sensing technology can better serve biodiversity conservation and assessment from different temporal and spatial scales with the development of remote sensing platforms and the improvement of sensors.

Key words: satellite remote sensing; airborne remote sensing; near-surface remote sensing; UAV; lidar

\section{前言}

生物多样性是多样化生命实体表现出的特征, 包括所有植物、动物、微生物以及所有的生态系统 及其形成的各种生态过程(马克平, 1993)。由于人类 活动加剧以及全球气候变暖, 全球物种正以前所未 有的速度丧失, 地球即将进入 “第六次生物大灭绝” 时代(Ceballos et al, 2015)。生物多样性保护和可持 续利用成为了全球关注的热点问题, 为了缓解物种 灭绝速率和理解生物多样性丧失机制, 生物学家、 生态学家以及政府和民众都致力于生物多样性的 研究和保护。中国是世界上生物多样性最丰富的 12 个国家之一, 世界四大遗传资源起源中心之一, 但 同时也是生物多样性受威胁最严重的国家之一。生 物多样性不仅是国内科研人员关注的热点问题, 而 且还深受党中央、国务院重视。

生物多样性描述自然界多样化程度, 是时间和 空间的函数(马克平, 1993), 描述生物多样性离不开 生物多样性监测。生物多样性监测主要在物种和生 态系统尺度上开展, 通常在典型植被类型区域建立 一定面积的长期固定样地, 对样地内部的物种组 成、结构、功能以及关键物种、濒危物种进行观测。 中国科学院生物多样性委员会于 2004年开始组织 有关研究所的科研人员和院外相关单位的合作者 共同建设中国森林生物多样性监测网络(CForBio), 通过布设大型固定样地对中国主要地带性森林类 型的生物多样性变化进行了连续监测, 在生物多样 性研究和保护方面做出了重要贡献。生物多样性监 测也是国际公约的重要履约内容, 如《生物多样性 公约》和《二十一世纪议程》等。与基础理论研究 相比, 国际公约更强调的是国家、区域和全球尺度 的生物多样性监测信息以及相关评估结果。在联合
国《生物多样性公约》爱知目标(2011-2020年生物 多样性战略规划)的推动下, 国家、区域到全球尺度 都在加强生物多样性监测工作, 以期为生物多样性 保护及其进展评估提供详实可靠的数据。

虽然传统的生物多样性监测和保护工作已经 取得了许多重要进展, 但是这些研究主要还是基于 地面调查方法, 重点关注物种和样地水平(Myers et al, 2000; Duro et al, 2007; 朱超等, 2015)。物种和样 地尺度上的调查结果在过去相当长一段时间内为 物种丰富度和均匀度评估提供了准确的信息, 但是 无法满足景观尺度、区域尺度甚至全球尺度上必要 的生物多样性监测需求(Nicholson et al, 2009)。生命 行星指数(living planet index, LPI)是世界自然基金 会(World Wildlife Fund, WWF)于1997年提出的一个 监测全球生物多样性状况的指标, 主要利用样点观 测的时间序列数据计算生活在陆地、淡水和海洋生 态系统中脊椎动物种群数量的平均变化速率。Loh 等(2005)利用生命行星指数对全球 1,100 个物种的 3,000 个种群的时间动态进行了分析, 发现 1970-2000年间陆地、淡水和海洋中的脊椎动物种 群数量减少了 $40 \%$, 其中淡水脊椎动物种群减少量 高达 $50 \%$, 陆地动物减少则相对较低, 平均下降 $25 \%$ 。然而受观测数据的限制, 这一指标仅能反映 脊椎动物的变化趋势, 还无法用于其他生物类群, 而且地面样点数据的采集需要大量人力和物力, 限 制了该指数的应用。另外, 在评估和预测全球变化 和人类活动影响下的生物多样性格局动态、揭示其 响应机制以及探究生物多样性与生态系统稳定性 关系等方面, 仅靠样点的监测结果很难有效地推动 决策层面保护措施的制定。

遥感作为获取生物多样性信息的另一种手段, 近年来在生物多样性领域发展迅速(图 1)。遥感数据 


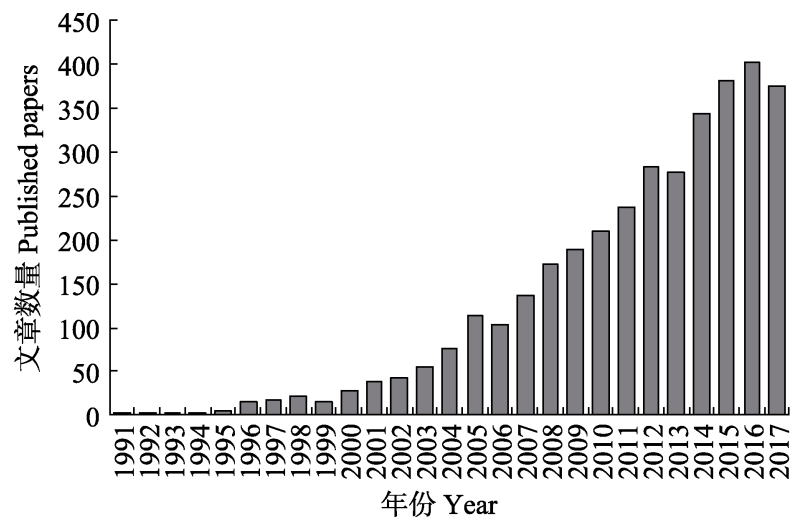

图1 遥感在生物多样性研究中的文献数量变化趋势

Fig. 1 The trend of literature quantity of remote sensing in biodiversity

覆盖广、序列性以及可重复等特点使其在大尺度生 物多样性监测、制图和评估方面具有极大优势 (Roughgarden et al, 1991)。利用遥感数据开展生物 多样性研究最早可以追溯到 20 世纪 80 年代, Löffler 和 Margules (1980)利用 60 米的 Landsat MSS 寻找袋 熊窝形成的草地秃块，从而监测了澳大利亚 Nullarbor 平原的袋熊。早期的生物多样性遥感监测 以星载和机载的影像数据为主, 侧重于土地利用和 土地覆盖的变化检测, 揭示土地利用方式变化带来 的生境斑块化和破碎化所导致的生物多样性降低

(Skole \& Tucker, 1993; Ramankutty \& Foley, 1999)。 伴随着高时空分辨率和光谱分辨率传感器以及新 型遥感平台的出现, 遥感观测已涵盖区域-洲际-全 球等多尺度、植物和动物等多领域的生物多样性监 测。而这些遥感平台在生物多样性研究中的应用进 展如何, 技术瓶颈怎样, 仍不清晰。因此, 本文通过 文献收集整理，从观测手段、研究尺度、观测对象、 生物多样性关注点等几方面综述不同遥感平台在 生物多样性研究中的应用现状, 分析不同遥感平台 的技术优势和局限性, 探讨未来遥感在生物多样性 研究中的应用趋势。

\section{2 不同遥感平台在生物多样性研究中的应用文}

遥感通常主要采用直接和间接两种途径来获 取生物多样性信息(Turner et al, 2003)。直接法是直 接识别物种或群落类型及其分布、多度, 这类方法 对遥感数据的空间分辨率和光谱分辨率有相当高 的要求。间接法是通过遥感数据衍生一些指标或变
量, 然后与野外观测数据结合构建模型预测生物多 样性。这些指标或变量被认为或证实是与生物多样 性密切相关，例如归一化植被指数 (Normalized Difference Vegetation Index, NDVI)、增强植被指数 (Enhanced Vegetation Index, EVI)等。

遥感平台按观测高度可分为卫星遥感、航空遥 感和近地面遥感(图2), 不同的高度意味着能够观测 到的空间尺度不同。通过ISI Web of Science数据库 搜索遥感在生物多样性应用方面的文献, 篮选关键 字和摘要发现：3种遥感平台中卫星遥感使用最多, 大约有 1,040 篇相关文章; 航空遥感大约有 43 篇相 关文章; 而近几年发展迅速的近地面遥感则有78篇 文章(图3a)。这些文献中，研究“洲际-全球”尺度的 文章大概有 439篇，景观-区域尺度的文章有530篇， 样地-景观尺度的文章有192篇(图3b)。从研究对象 的角度来看, 利用遥感手段开展植物多样性的研究 最多，共578篇; 其次则是通过获取生境信息来开 展生物多样性研究, 共399篇; 开展动物类群研究 较少, 其中鸟类和大型哺乳动物研究相对多些, 分 别为76篇和54篇，水生动物和浮游生物分别为 23 篇 和20篇; 微生物类的研究只查询到2篇。

遥感平台常用的传感器包括三类：光学传感 器、微波雷达传感器和激光雷达传感器。光学传感 器包括高分相机、多光谱成像仪、高光谱成像仪和 热成像仪，分别获取特定波段范围内的光谱信息。

其中, 高分相机和多光谱成像仪可以获取植物的颜 色信息和纹理特征; 高光谱成像仪获取的影像可用 于反演植物生化组分; 热成像仪可以提取地物的温 度信息; 激光雷达遥感传感器能获得精细的地物三 维信息。通过文献整理发现：高分相机和多光谱/高 光谱成像仪在生物多样性研究中使用最多(图4), 新 兴的激光雷达传感器的使用增长十分迅速, 而热红 外成像仪只有少许应用。

\section{1 卫星遥感}

自从美国宇航局共享Landsat系列卫星数据以 来, 卫星遥感为生态学领域的各类研究提供了观测、 分析和预测的数据(Turner et al, 2003; Pettorelli et al, 2014)。相比于地面观测, 卫星遥感具有以下几点优 势: (1)观测范围大, 能够直接获取全球尺度的数据; (2)能够实现长时间序列的观测和记录; (3)数据类型 多样, 数据获取成本低。这些优势有利于生态学家 


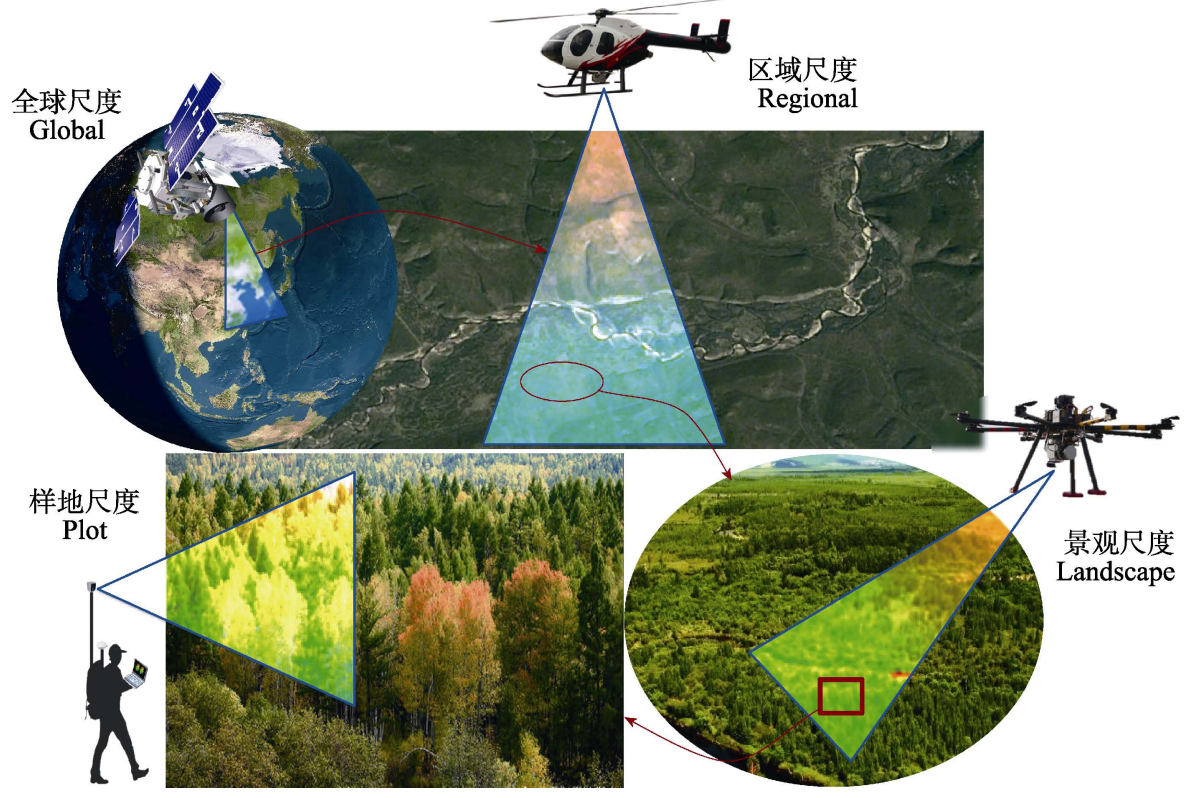

图2 不同遥感平台在生物多样性研究中的观测尺度

Fig. 2 Observation scale of different remote sensing platforms in biodiversity research
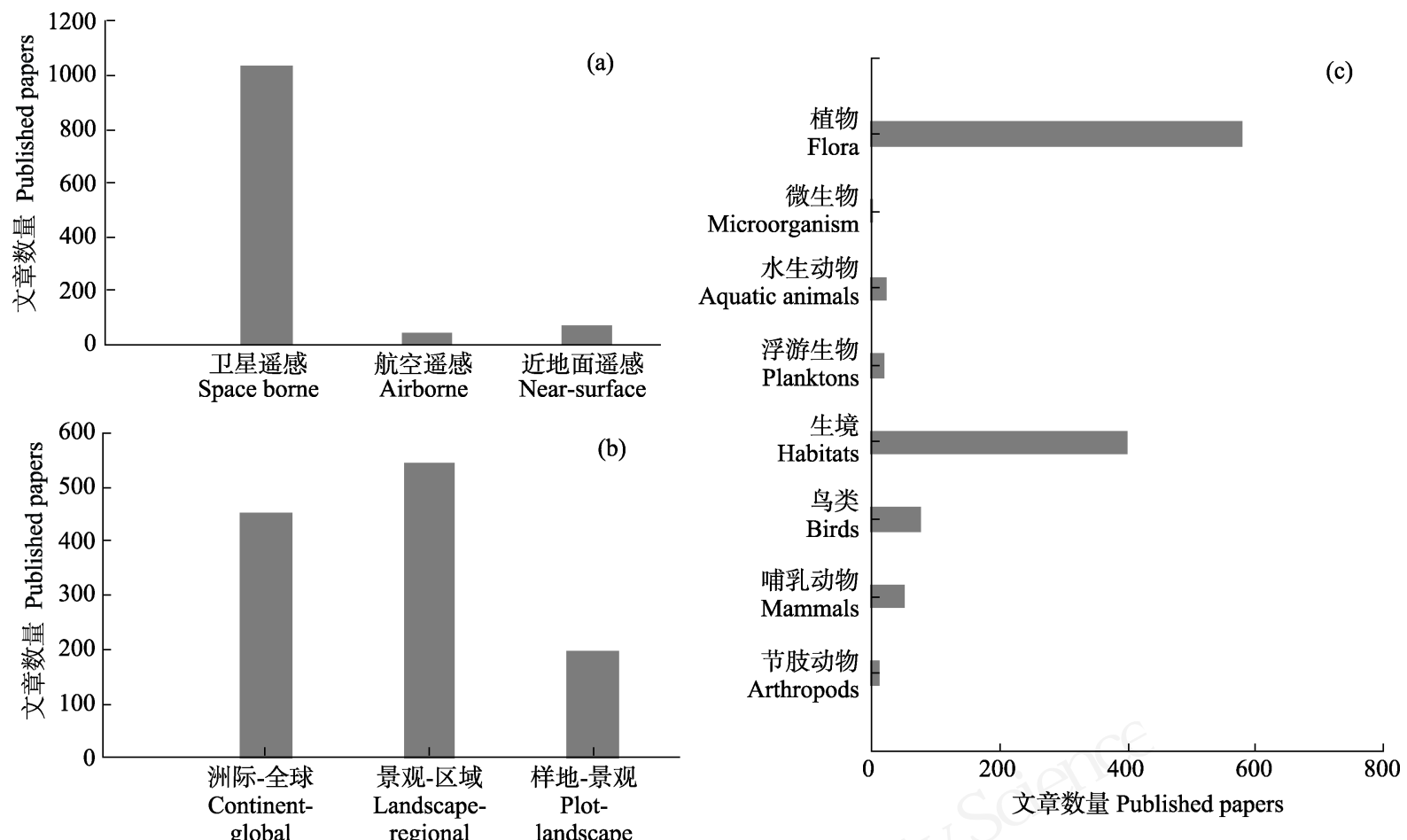

图3 生物多样性研究在遥感平台(a)、关注尺度(b)和研究对象(c)中的文献数量

Fig. 3 Literature quantity of remote sensing platform (a), study scale (b) and biodiversity group (c) in biodiversity research

获取物种组成、生态系统结构和功能相关信息, 尤 其是长期积累的多源遥感数据为大尺度和长时间序 列的生物多样性监测提供了可能(Kerr \& Ostrovsky, 2003; Gillespie et al, 2008; Paganini et al, 2016)。
历经几十年的发展, 遥感卫星已形成成熟的观 测体系。现有卫星在观测和记录电磁信号方面各有 不同, 其能够获取的目标信息也有所不同(表1)。遥 感卫星分辨率从公里级逐渐迈入米级时代，如 


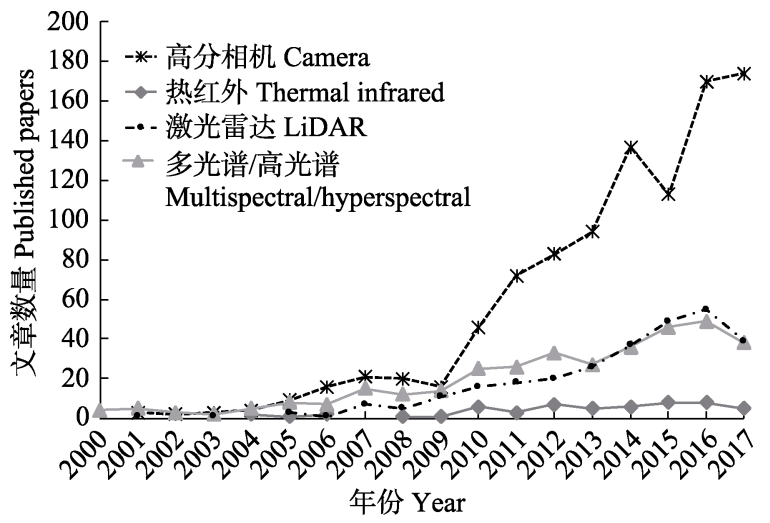

图4 不同传感器在生物多样性研究中的文献数量变化趋势 Fig. 4 The trend of literatures about different remote sensing senors using in biodiversity research
IKONOS，OrbView及RapidEye等卫星。2000年后， 大量的商用陆地资源遥感卫星进入亚米级时代, 如 QuickBird等。随着卫星时空光谱分辨率的不断提升, 大量学者利用卫星遥感技术获取与生物多样性相 关的信息, 开展生物多样性保护评估和监测的研究 (Gould, 2000; Kerr et al, 2001; Rocchini et al, 2007; Saatchi et al, 2008; Paganini et al, 2016)。

目前, 卫星遥感数据主要用于植物和动物多样 性研究工作中, 其中在植物多样性研究方面, 以卫 星遥感为数据基础的研究主要关注物种组成、生态 系统结构和功能三个方面(Martin et al, 1998; Carleer \& Wolff, 2004)。在研究物种组成方面, Saatchi

表1 国内外常用遥感卫星及其基本参数简介

Table 1 the parameters list of Chinese and international popular remote sensing satellites

\begin{tabular}{|c|c|c|c|c|c|c|}
\hline $\begin{array}{l}\text { 卫星 } \\
\text { Satellite }\end{array}$ & $\begin{array}{l}\text { 传感器 } \\
\text { Sensor }\end{array}$ & $\begin{array}{l}\text { 传感器类型 } \\
\text { Type of sensor }\end{array}$ & $\begin{array}{l}\text { 波段数 } \\
\text { Bands }\end{array}$ & $\begin{array}{l}\text { 空间分辨率 } \\
\text { Spatial resolution }\end{array}$ & $\begin{array}{l}\text { 重返时间 } \\
\text { Repeat } \\
\text { interval }\end{array}$ & $\begin{array}{l}\text { 发射时间 } \\
\text { Launch date }\end{array}$ \\
\hline LandSat 5 & $\mathrm{TM}$ & $\begin{array}{l}\text { 多光谱 } \\
\text { Multispectral }\end{array}$ & 7 & $\begin{array}{l}\text { Band 1-5, 7: } 30 \mathrm{~m} \\
\text { Band 6: } 120 \mathrm{~m}\end{array}$ & $16 \mathrm{~d}$ & $\begin{array}{l}1984 \\
\text { (2013 宣布失效) } \\
\text { (Deactivated in 2013) }\end{array}$ \\
\hline LandSat 7 & ETM+ & $\begin{array}{l}\text { 全色/多光谱 } \\
\text { Panchromatic/ } \\
\text { multispectral }\end{array}$ & 8 & $\begin{array}{l}\text { Band 8: } 15 \mathrm{~m} \\
\text { Band 1-5, 7: } 30 \mathrm{~m} \\
\text { Band 6: } 60 \mathrm{~m}\end{array}$ & $16 \mathrm{~d}$ & $\begin{array}{l}1999 \\
\text { (2003.05 设备故障, 影 } \\
\text { 像出现条带状) } \\
\text { (SLC-off in 2003.05) }\end{array}$ \\
\hline \multirow[t]{2}{*}{ LandSat 8} & OLI & $\begin{array}{l}\text { 全色/多光谱 } \\
\text { Panchromatic/ } \\
\text { multispectral }\end{array}$ & 9 & $\begin{array}{l}\text { Band 8: } 15 \mathrm{~m} \\
\text { Band 1-7, 9: } 30 \mathrm{~m}\end{array}$ & $16 \mathrm{~d}$ & 2013 \\
\hline & TRS & $\begin{array}{l}\text { 热红外 } \\
\text { Thermal infrared }\end{array}$ & 2 & $100 \mathrm{~m}$ & & \\
\hline IKONOS & $\begin{array}{l}\text { CCD相机 } \\
\text { CCD camera }\end{array}$ & $\begin{array}{l}\text { 全色/多光谱 } \\
\text { Panchromatic/ } \\
\text { multispectral }\end{array}$ & 4 & $\begin{array}{l}\text { 全色: } 1 \mathrm{~m} \\
\text { Panchromatic } \\
\text { 多光谱: } 4 \mathrm{~m} \\
\text { Multispectral }\end{array}$ & $3 \mathrm{~d}$ & $\begin{array}{l}1999 \\
\text { (2015年宣布退役) } \\
\text { (Deactivated in 2015) }\end{array}$ \\
\hline SPOT 5 & HRG & $\begin{array}{l}\text { 全色/多光谱 } \\
\text { Panchromatic/ } \\
\text { multispectral }\end{array}$ & 4 & $\begin{array}{l}\text { 全色: } 2.5 \mathrm{~m} \\
\text { Panchromatic } \\
\text { Band 1-3: } 10 \mathrm{~m} \\
\text { Band 4: } 20 \mathrm{~m}\end{array}$ & $26 \mathrm{~d}$ & 2002 \\
\hline SPOT 7 & NAOMI & $\begin{array}{l}\text { 全色/多光谱 } \\
\text { Panchromatic/ } \\
\text { multispectral }\end{array}$ & 4 & $\begin{array}{l}\text { 全色: } 1.5 \mathrm{~m} \\
\text { Panchromatic } \\
\text { 多光谱: } 6 \mathrm{~m} \\
\text { Multispectral }\end{array}$ & $26 \mathrm{~d}$ & 2014 \\
\hline WorldView-3 & CCD相机 & $\begin{array}{l}\text { 全色/多光谱/短波红外 } \\
\text { /CAVIS } \\
\text { Panchromatic/ } \\
\text { multispectral/ } \\
\text { Short wavelength } \\
\text { infrared/CAVIS }\end{array}$ & 29 & $\begin{array}{l}\text { 全色: } 0.31 \mathrm{~m} \\
\text { Panchromatic } \\
\text { 多光谱: } 1.24 \mathrm{~m} \\
\text { Multispectral } \\
\text { 短波红外: } 3.7 \mathrm{~m} \\
\text { Short wavelength infrared } \\
\text { CAVIS } 30 \mathrm{~m}\end{array}$ & 小于 $1 \mathrm{~d}$ & 2014 \\
\hline
\end{tabular}


表1 (续) Table 1 (continued)

\begin{tabular}{|c|c|c|c|c|c|c|}
\hline $\begin{array}{l}\text { 卫星 } \\
\text { Satellite }\end{array}$ & $\begin{array}{l}\text { 传感器 } \\
\text { Sensor }\end{array}$ & $\begin{array}{l}\text { 传感器类型 } \\
\text { Type of sensor }\end{array}$ & $\begin{array}{l}\text { 波段数 } \\
\text { Bands }\end{array}$ & $\begin{array}{l}\text { 空间分辨率 } \\
\text { Spatial resolution }\end{array}$ & $\begin{array}{l}\text { 重返时间 } \\
\text { Repeat } \\
\text { interval }\end{array}$ & $\begin{array}{l}\text { 发射时间 } \\
\text { Launch date }\end{array}$ \\
\hline WorldView-4 & CCD相机 & $\begin{array}{l}\text { 全色/多光谱 } \\
\text { Panchromatic/ } \\
\text { multispectral }\end{array}$ & 4 & $\begin{array}{l}\text { 全色: } 0.31 \mathrm{~m} \\
\text { Panchromatic } \\
\text { 多光谱: } 1.24 \mathrm{~m} \\
\text { Multispectral }\end{array}$ & 1或 $4.5 \mathrm{~d}$ & 2016 \\
\hline \multirow[t]{2}{*}{ Terra } & ASTER & $\begin{array}{l}\text { 近红外/短波红外/ } \\
\text { 热红外 } \\
\text { Near Infrared/Short } \\
\text { Wavelength Infrared/ } \\
\text { Thermal Infrared }\end{array}$ & 15 & $\begin{array}{l}\text { 近红外: } 15 \mathrm{~m} \\
\text { Near Infrared } \\
\text { 短波红外: } 30 \mathrm{~m} \\
\text { Short wavelength infrared } \\
\text { 热红外: } 90 \mathrm{~m} \\
\text { Thermal infrared }\end{array}$ & $16 \mathrm{~d}$ & 1999 \\
\hline & MODIS & $\begin{array}{l}\text { 多光谱 } \\
\text { Multispectral }\end{array}$ & 36 & $\begin{array}{l}\text { Band 1, 2: } 250 \mathrm{~m} \\
\text { Band 3-7: } 500 \mathrm{~m} \\
\text { Band 8-36: } 1,000 \mathrm{~m}\end{array}$ & & \\
\hline AQUA & MODIS & $\begin{array}{l}\text { 多光谱 } \\
\text { Multispectral }\end{array}$ & 36 & $\begin{array}{l}\text { Band 1, 2: } 250 \mathrm{~m} \\
\text { Band 3-7: } 500 \mathrm{~m} \\
\text { Band 8-36: } 1,000 \mathrm{~m}\end{array}$ & $16 \mathrm{~d}$ & 2002 \\
\hline Sentinel-1A & $\begin{array}{l}\text { C波段合成孔径雷达 } \\
\text { SAR (synthetic aperture } \\
\text { radar) with C band }\end{array}$ & SAR & - & $\begin{array}{l}\text { 条带模式:5*5 m } \\
\text { Strip map mode } \\
\text { 干涉宽幅模式: } 5 * 20 \mathrm{~m} \\
\text { Interferometric wide swath } \\
\text { mode } \\
\text { 超宽幅模式: } 20 * 40 \mathrm{~m} \\
\text { Extra wide swath mode }\end{array}$ & $6 \mathrm{~d}$ & 2014 \\
\hline Sentinel-2A & $\begin{array}{l}\text { 多光谱成像仪 } \\
\text { Multispectral scanner }\end{array}$ & $\begin{array}{l}\text { 多光谱 } \\
\text { Multispectral }\end{array}$ & 13 & $\begin{array}{l}\text { Band 2-4, 8: } 10 \mathrm{~m} \\
\text { Band 5-7, 8A, 11, 12: } 20 \mathrm{~m} \\
\text { Band 1, 9, 10: } 60 \mathrm{~m}\end{array}$ & $10 \mathrm{~d}$ & 2015 \\
\hline \multirow[t]{2}{*}{ GF-1 } & $\begin{array}{l}\text { 全色多光谱相机 } \\
\text { Panchromatic multispectral } \\
\text { scanner }\end{array}$ & $\begin{array}{l}\text { 全色/多光谱 } \\
\text { Panchromatic/ } \\
\text { multispectral }\end{array}$ & 5 & $\begin{array}{l}\text { Band 1: } 2 \mathrm{~m} \\
\text { Band 2-5: } 8 \mathrm{~m}\end{array}$ & $4 \mathrm{~d}$ & 2013 \\
\hline & $\begin{array}{l}\text { 多光谱相机 } \\
\text { Multispectral scanner }\end{array}$ & $\begin{array}{l}\text { 多光谱 } \\
\text { Multispectral }\end{array}$ & 4 & $16 \mathrm{~m}$ & & \\
\hline GF-2 & $\begin{array}{l}\text { 全色多光谱相机 } \\
\text { Panchromatic multispectral } \\
\text { scanner }\end{array}$ & $\begin{array}{l}\text { 全色/多光谱 } \\
\text { Panchromatic/ } \\
\text { multispectral }\end{array}$ & 5 & $\begin{array}{l}\text { Band 1: } 1 \mathrm{~m} \\
\text { Band 2-5: } 4 \mathrm{~m}\end{array}$ & $5 \mathrm{~d}$ & 2014 \\
\hline GF-3 & $\begin{array}{l}\text { C波段合成孔径雷达 } \\
\text { SAR with C band }\end{array}$ & SAR & - & $\begin{array}{l}\text { 因成像模式而定 } \\
(1,3,5,8,10,25,50,100, \\
500 \mathrm{~m}) \\
\text { Resolution depend on the } \\
\text { scan mode }\end{array}$ & - & 2016 \\
\hline GF-4 & $\begin{array}{l}\text { 面阵凝视相机 } \\
\text { Staring array camera }\end{array}$ & $\begin{array}{l}\text { 可见光/近红外/ } \\
\text { 中波红外 } \\
\text { Visible/Near infrared/ } \\
\text { Middle infrared }\end{array}$ & 6 & $\begin{array}{l}\text { 可见光近红外: } 50 \mathrm{~m} \\
\text { Visible/near infrared } \\
\text { 中波红外: } 400 \mathrm{~m} \\
\text { Middle infrared }\end{array}$ & $20 \mathrm{~s}$ & 2015 \\
\hline \multirow[t]{2}{*}{ ZY-1 02C } & $\begin{array}{l}\text { 全色多光谱相机 } \\
\text { Panchromatic multispectral } \\
\text { scanner }\end{array}$ & $\begin{array}{l}\text { 全色/多光谱 } \\
\text { Panchromatic/ } \\
\text { multispectral }\end{array}$ & 4 & $\begin{array}{l}\text { 全色: } 5 \mathrm{~m} \\
\text { Panchromatic } \\
\text { 多光谱: } 10 \mathrm{~m} \\
\text { Multispectral }\end{array}$ & $3 \mathrm{~d}$ & 2011 \\
\hline & $\begin{array}{l}\text { 全色高分辨率相机 } \\
\text { Panchromatic } \\
\text { high-resolution scanner }\end{array}$ & $\begin{array}{l}\text { 全色 } \\
\text { Panchromatic }\end{array}$ & - & $2.36 \mathrm{~m}$ & & \\
\hline \multirow[t]{3}{*}{ ZY-3 } & $\begin{array}{l}\text { 正视全色TDI CCD相机 } \\
\text { Ortho-panchromatic TDI } \\
\text { CCD camera }\end{array}$ & $\begin{array}{l}\text { 全色 } \\
\text { Panchromatic }\end{array}$ & - & $2.1 \mathrm{~m}$ & $5 d$ & 2012 \\
\hline & $\begin{array}{l}\text { 前视、后视TDI CCD相机 } \\
\text { fore sigh and back sight TDI } \\
\text { CCD camera }\end{array}$ & $\begin{array}{l}\text { 全色 } \\
\text { Panchromatic }\end{array}$ & - & $3.5 \mathrm{~m}$ & & \\
\hline & $\begin{array}{l}\text { 正视多光谱相机 } \\
\text { Multispectral ortho-imager }\end{array}$ & $\begin{array}{l}\text { 多光谱 } \\
\text { Multispectral }\end{array}$ & 4 & $6 \mathrm{~m}$ & & \\
\hline
\end{tabular}




\begin{tabular}{|c|c|c|c|c|c|c|}
\hline & & & & & & \\
\hline $\begin{array}{l}\text { 卫星 } \\
\text { Satellite }\end{array}$ & $\begin{array}{l}\text { 传黛器 } \\
\text { Sensor }\end{array}$ & $\begin{array}{l}\text { 传柖器尜型 } \\
\text { Type of sensor }\end{array}$ & $\begin{array}{l}\text { 波段数 } \\
\text { Bands }\end{array}$ & $\begin{array}{l}\text { 空间分辨率 } \\
\text { Spatial resolution }\end{array}$ & $\begin{array}{l}\text { 重返时间 } \\
\text { Repeat } \\
\text { interval }\end{array}$ & $\begin{array}{l}\text { 发射时间 } \\
\text { Launch date }\end{array}$ \\
\hline \multirow[t]{2}{*}{ HJ-1A } & $\begin{array}{l}\text { CCD相机 } \\
\text { CCD camera }\end{array}$ & $\begin{array}{l}\text { 多光谱 } \\
\text { Multispectral }\end{array}$ & 4 & $30 \mathrm{~m}$ & $4 \mathrm{~d}$ & 2008 \\
\hline & $\begin{array}{l}\text { 高光谱成像仪 } \\
\text { Hypperspectral scanner }\end{array}$ & $\begin{array}{l}\text { 高光谱 } \\
\text { Hyperspectral }\end{array}$ & $110-128$ & $100 \mathrm{~m}$ & & \\
\hline \multirow[t]{2}{*}{ HJ-1B } & $\begin{array}{l}\text { CCD相机 } \\
\text { CCD camera }\end{array}$ & $\begin{array}{l}\text { 多光谱 } \\
\text { Multispectral }\end{array}$ & 4 & $30 \mathrm{~m}$ & $4 \mathrm{~d}$ & 2008 \\
\hline & $\begin{array}{l}\text { 红外多光谱相机 } \\
\text { Infrared multispectral } \\
\text { scanner }\end{array}$ & $\begin{array}{l}\text { 红外 } \\
\text { Infrared }\end{array}$ & 4 & $150 \mathrm{~m}$ & & \\
\hline HJ-1C & $\begin{array}{l}\text { 合成孔径雷达 } \\
\text { SAR }\end{array}$ & SAR & - & $\begin{array}{l}\text { 单视模式: } 5 \mathrm{~m} \\
\text { Single mode } \\
\text { 距离向四视模式: } 20 \mathrm{~m} \\
\text { Four sights at range } \\
\text { direction }\end{array}$ & $4 \mathrm{~d}$ & 2012 \\
\hline
\end{tabular}

等(2008)利用多源遥感数据, 结合与植被和气候相 关的环境变量信息, 利用最大熵模型预测了亚马逊 流域5种树的分布和多样性特征; Wilson等(2012)利 用森林清查数据和中分辨率的遥感影像数据 (Moderate Resolution Imaging Spectroradiometer, MODIS)研究了大尺度树种组成和分布。然而, 利用 卫星遥感开展物种组成研究存在很大的局限性, 很 多研究是建立在海量样本的基础上, 例如Wilson等 (2012)的研究使用的是美国森林清查数据, 而且遥 感数据只是提供了物候的信息。Saatchi等(2008)的 研究则是分析部分树种的潜在分布, 与真实的物种 分布和组成存在很大差异。在生态系统结构多样性 方面, 利用光学影像获取光谱指数反演物种性状是 最常用的手段之一(Clerici et al, 2012; Duffy \& Pettorelli, 2012), 但受光学遥感的饱和效应影响, 其精 度都很低。近年来, 激光雷达、合成孔径雷达技术 等主动遥感技术的发展, 为精确测量森林三维结构 (如树高、叶面积指数等)、预测生物量等提供了全 新的手段(Hu et al, 2016; Su et al, 2016a, 2017)。在生 态系统功能方面, 主要是利用时间序列的遥感影像 监测植被动态变化及其对气候变化的响应等 (Jordan et al, 2012; Hutchinson et al, 2015; Lausch et $\mathrm{al}, 2016)$ 。利用卫星研究植被动态通常关注植被类 型的变化, 即生态系统多样性, 对于物种尺度的变 化研究较少。

在动物多样性研究方面, 卫星遥感多以生境的 探测为主, 进而分析和预测动物的分布、迁徙 (Austin et al, 1996; Handcock et al, 2009)。如Luschi
等(1998)通过卫星观测的手段研究了海龟迁徙的路 线, 发现其在长距离迁徙过程中尽管受到外部因素 的干扰，仍能够始终保持朝着目的地方向前进; Kerr等(2001)利用遥感卫星获取的土地覆盖信息， 通过分析蝴蝶栖息地不同特征，成功预测了加拿大 各地区蝴蝶的物种丰富度和群落相似度。受限于卫 星数据的空间分辨率和时间分辨率，大部分卫星遥 感数据不能直接观测到动物的个体, 而且由于动物 的习性不同, 不同物种对于生境要素的要求也不一 致, 而且影响因素比较复杂, 无法像反演植物多样 性一样找到类似的要素。

此外, 卫星遥感指标还常用于构建生物多样性 指标, 用于评估区域和全球尺度的生物多样性现状 和变化趋势。Coops等(2008)利用 MODIS反演的 fPAR产品, 从生态系统的能量角度出发, 按照食物 供给能力、越冬时期供给能力和食物供给稳定性三 个维度构建了动态生境指数(Dynamic Habitat Index, DHI), 用于监测和评估大尺度的生物多样性现状和 变化。虽然各国都开展了大量关于生物多样性的研 究, 但是生态学领域和遥感领域的研究人员缺少交 流, 生物多样性观测数据存在获取不充分、数据不 连续等问题, 从而导致监测内容和评价指标缺乏一 致性, 不利于在区域和全球尺度上不同生态系统之 间开展标准的生物多样性监测和管理(Skidmore \& Pettorelli, 2015)。为了能够及时获取周期性的、全 球观测的标准数据, 全球生物多样性观测网络 (Group on Earth Observations Biodiversity Observation Network, GEO BON)的科学家们提出了生物多 
样性核心指标(Essential Biodiversity Variables, EBV) 的概念。EBV从 6 个方面描述生物多样性: 遗传组 成、物种数量、物种性状、群落组成、生态系统功 能和生态系统结构(Pereira et al, 2013)。除了遗传组 成之外, 其他类别都能够通过用遥感的手段获取 (Skidmore \& Pettorelli, 2015)。其中, 生态系统结构 和功能可通过卫星遥感直接观测, 物种数量、物种 性状和群落组成则难以通过卫星遥感直接观测 (表2)。

\section{2 航空遥感}

与卫星遥感固定轨道和过境周期相比, 航空遥 感理论上可在所需的任何时间进行遥感数据的获 取。航空遥感通常集成多传感器, 单次观测即可获 取不同类型的遥感数据, 便于后期多传感器数据的 配准、融合处理。

航空遥感兼顾了不同尺度水平的数据获取和 分析能力, 尤其在景观-区域尺度有着不可比拟的 优势, 并广泛应用于生态系统组成、结构和功能研 究。在植物多样性研究领域, 主要集中在植被性状 (Asner et al, 2017)、植被结构(Coops et al, 2016)、物 种识别(Asner \& Martin, 2008; Sasaki et al, 2012)以 及物种多样性(Carlson et al, 2007; Simonson et al, 2012)等方面。如Carlson等(2007)分析了夏威夷17个 森林站点的林冠维管束植物丰富度与高光谱反射 率的关系, 并指出与生化组分相关的几个波段特征 能够很好地指示物种多样性。Simonson等(2012)利 用机载激光雷达获取的冠层结构信息(如植被高度) 来衡量物种组成和生物多样性; Coops等(2016)利用 机载激光雷达能够精确刻画森林三维结构的优势,
研究了物种分布与森林生境结构的关系, 并提出了 高度、覆盖度和复杂度三个森林水平和垂直结构的 指数用于表征森林生物多样性水平。航空遥感通常 搭载多种传感器, 一次航飞即可获取多类型数据, 进行数据融合后可更好地提取生物多样性信息。Su 等(2016b)在美国加州内华达山脉开展了基于机载 激光雷达和影像的植被类型制图研究，利用贝叶斯 和K-均值的自动聚类算法的非监督分类方法, 最终 发现融入激光雷达数据的分类效果相比于只有光 学影像的分类方法更加精确, 能够识别的植被类型 更多。Asner等(2017)利用机载激光雷达和高光谱数 据获取森林冠层的性状特征, 结合地形气候等环境 分析了森林功能多样性的变化。Zhao等(2018)在神 农架利用机载激光雷达和高光谱数据, 通过对植物 结构特征和生化组分特征聚类来估算生物多样性。

目前, 航空遥感很难获取准确的物种信息来反映生 物多样性, 而且很多研究反演的物种多样性只是性 状特征的聚类，无法与真实的物种对应而且容易出 现饱和现象。

机载激光雷达的出现很大程度上促进了遥感 在动物多样性领域研究的发展(Davies \& Asner, 2014), 如飞行的脊椎动物(鸟类与蝙蝠) (Goetz et al, 2010, 2014)、非飞行的脊椎动物(食鱼貂与鹿) (Zhao et al，2012)、无脊椎动物(甲虫与蜘蛛) (Müller \& Brandl, 2009)以及其他类别动物的研究。机载激光 雷达通过刻画精细的三维生境信息，根据地面观测 分析和提取动物的栖息地特征，从而开展动物空间 分布、种群密度和动物保护等研究(Clawges et al, 2008; Purkis et al, 2008)。Goetz等(2010)利用激光雷

表2 卫星遥感能够获取的与生物多样性核心指标相关的指标

Table 2 Biodiversity indicators that can be derived from satellite remote sensing

\begin{tabular}{|c|c|}
\hline $\begin{array}{l}\text { 生物多样性核心指标 } \\
\text { Essential biodiversity } \\
\text { variables }\end{array}$ & $\begin{array}{l}\text { 卫星遥感能获取的指标 } \\
\text { Indicators obtained from satellite remote sensing }\end{array}$ \\
\hline $\begin{array}{l}\text { 物种数量 } \\
\text { Species populations }\end{array}$ & 物种分布 Species distribution (Saveraid et al, 2001; Leyequien et al, 2007; Shirley et al, 2013; Sequeira et al, 2014) \\
\hline $\begin{array}{l}\text { 物种性状 } \\
\text { Species traits }\end{array}$ & 叶面积指数 LAI (Fuentes et al, 2008)、氮素含量 Nitrogen content (Ojoyi et al, 2017)等 \\
\hline $\begin{array}{l}\text { 群落组成 } \\
\text { Community composition }\end{array}$ & $\begin{array}{l}\text { 物种密度 Species density (Hernández-Stefanoni \& Dupuy, 2007; Godinho et al, 2016)、物种丰富度 Species richness } \\
\text { (Knudby et al, 2010; Lucas et al, 2010)等 }\end{array}$ \\
\hline $\begin{array}{l}\text { 生态系统功能 } \\
\text { Ecosystem function }\end{array}$ & $\begin{array}{l}\text { 植被绿度 Greenness (Zhou et al, 2014)、植被物候 Phenology (Ganguly et al, 2010)、光合作用能力和生态系统生产力 } \\
\text { Photosynthesis and ecosystem productivity (Franklin et al, 2006; Mishra \& Chaudhuri, 2015)等 }\end{array}$ \\
\hline $\begin{array}{l}\text { 生态系统结构 } \\
\text { Ecosystem structure }\end{array}$ & $\begin{array}{l}\text { 景观破碎化和异质性 Landscape fragmentation and heterogeneity (Saatchi et al, 2001; Wang \& Moskovits, 2001; } \\
\text { Tuanmu \& Jetz, 2015)、土地覆盖和土地利用 Landcover and land use (Farashi et al, 2016; Zhao et al, 2016)、植被高度 } \\
\text { Vegetation height (Su et al, 2017)等 }\end{array}$ \\
\hline
\end{tabular}


达数据反演的冠层垂直结构信息分析了一种热带 鸟类的分布特征并指出: 由激光雷达数据获得的森 林结构数据是确定鸟类多样性的关键参数。由此建 立了乌类丰富度与森林结构的关系模型, 成功预测 了该种鸟类在北美的分布情况(Goetz et al, 2014)。 Zhao等(2012)利用电子项圈监测食鱼貂的活动信息, 同时结合机载激光雷达获取的森林垂直结构信息, 揭示了食鱼貂对生境的喜好。Tempel等(2015)利用 机载激光雷达获取的覆盖度和大树密度分析出加 州斑点猫头鹰(Strix occidentalis)的筑巢特性, 并结 合林火发生前后的森林结构差异推断出林火发生 对猫头鹰产生的影响。Vihervaara 等(2015)结合地 面调查的鸟类栖息情况与机载激光雷达数据获得 的森林植被结构信息开展了景观尺度上的生物多 样性评估, 并证实机载激光雷达数据提取的大量结 构参数可用于量化生物多样性核心指标EBV。总而 言之, 机载激光雷达获取的大范围森林垂直结构信 息能够很好地用于动物多样性研究。如何从众多参 数中提出共性的指标用于动物多样性监测, 则是下 一步研究的关键。

\section{3 近地面遥感平台}

近地面遥感是近年来兴起的一个概念, 主要指 利用低空飞行的无人机、飞艇以及地面上固定或移 动的载具来获取遥感数据, 如塔台、脚架、汽车、 人等。与传统的卫星遥感和航空遥感相比, 近地面 遥感平台观测的尺度更贴近地面, 能够在地面观 测、航空遥感和卫星遥感之间搭建起信息推绎的可 靠桥梁。正因为近地面遥感的这个优势, 中国生物 多样性监测与研究网络在综合中心设了近地面遥 感平台, 用于获取观测网络中大样地的生境信息 (郭庆华等, 2016a)。

\subsection{1 无人机平台}

无人机是无人驾驶飞机(unmanned aerial vehicle, UAV)的简称。无人机具有灵活、高效、便捷的 特点, 克服了传统遥感技术在时间和空间分辨率上 的局限性, 同时也避开了云层遮挡的干扰(胡健波 和张健, 2018), 满足遥感动态观测需求和定制化遥 感需求; 同时能与地面调查数据更好地衔接, 使获 取大尺度精细数据成为可能(郭庆华等, 2016b)。无 人机平台之所以能够在生态遥感、资源环境保护、 生物多样性监测等领域得到大量应用, 主要原因在 于: (1)无人机很好地弥补了区域遥感监测与样方调
查之间的尺度空缺; (2)无人机硬件成本下降和稳定 性上升; (3)小型和轻量化的传感器出现; (4)无人机 平台数据后处理软件增多并成熟和商业化。目前, 在生物多样性的研究中常用多旋翼轻、微型无人机 采集遥感数据, 并依据研究需求有选择性地搭载相 机、光谱成像仪、激光雷达等传感器(郭庆华等, 2016b)。

无人机平台与卫星遥感、航空遥感平台一样能 够直接反演得到EBV指标中的生态系统功能和结 构信息(图5), 不同的是无人机平台能够直接观测到 物种的个体，从而能直接获取以前只能依靠回归方 式获取的物种数量、物种性状和群落组成等信息。 在森林生物多样性评估方面, Getzin等(2012)从无人 机相机获取的高分辨率影像中提取温带森林的林 窗信息, 结合实测数据估测了温带森林的生物多样 性。在物种分布方面, Kalacska等(2013)用旋翼无人 机的高分辨率影像对加拿大渥太华东部一个沼泽 的苔藓进行分类，使用该低成本非破坏性方法推算 出白毛羊胡子草(Eriophorum vaginatum)的覆盖率。 国内的学者也用无人机开展了一些探索研究, 如 Zhang等(2016)在鼎湖山国家自然保护区的20公顷 样地范围内使用无人机获取冠层信息, 证明无人机 可以为长期森林监测提供低成本、高分辨率数据。

无人机平台用于开展动物多样性研究具有独 特的优势, 尤其是大型动物的种群数量和动物保护 方面。Vermeulen等(2013)用无人机对非洲西部的非 洲象进行监测, 共监测到34头大象。Mulero-Pazmany等(2014)将无人机用作犀牛反偷猎的工具, 使其成为非洲生物多样性保护的利器。对于海洋动 物无人机也能发挥重要作用。研究者分别用无人机 对海豹(Krause et al, 2017)、海狮(Adame et al, 2017)、海龟 (Schofield et al, 2017)、儒艮 (Hodgson et $\mathrm{al}, 2013)$ 等海洋哺乳动物进行了监测。除了直接监 测动物的数据和分布, 无人机平台还能更细致地获 取生境信息用于各种动物的栖息地研究, 如黑猩猩 (Pongo abelii) (Szantoi et al, 2017)、鲨鱼(Carcharhinus melanopterus and Himantura fai) (Kiszka et al, 2016)、鳄鱼(Crocodylus porosus)(Evans et al, 2015)等。Sarda-Palomera等(2012)在无人机鸟类监测 研究中也已取得了明显的进展。此外还有人专门研 究了无人机监测对野生动物的干扰(Brisson-Curadeau et al, 2017; Mulero-Pazmany et al, 2017)。 


\subsection{2 地基平台}

地基平台依靠地面上固定或移动的物体作为 载具, 其载荷包括高分相机、热红外成像仪、多/高 光谱成像仪、地基激光雷达、背包激光雷达等。相 比与星载和航空平台, 地基平台的观测尺度最小, 但是其获取的数据精度更高, 而且能够获取其他遥 感平台难以准确获取的林下信息(图6)。

地基平台包括了一些传统的地面观测方法, 如 光学和红外相机, 也包含了一些新的遥感方式, 如 车载激光雷达、地基激光雷达、背包激光雷达等。 红外相机是一种红外触发拍摄技术 (infraredtriggered photography), 该技术利用红外感应设备在无 人现场操作的情况下自动拍摄野生动物的照片或 影像(李戟等, 2014), 在国内外兽类和鸟类的监测中 应用非常广泛, 尤其是对于一些有昼伏夜出习性的 物种监测。李生强等(2016)使用红外相机对喀斯特 生境兽类和鸟类多样性及活动节律进行监测, 共监 测到20种兽类和26种鸟类, 为广西弄岗国家级自然 保护区的管理提供了有效数据支持。除了红外相机, 现在很多野外台站在通量塔上放置光学相机搜集 研究区的照片和视频信息, 用于获取生态系统的物 候信息, 来分析物种组成与物候的关系。如Weil等 (2017)在地中海地区利用数码相机观测了来自12种 1,839 个地中海植物个体的物候, 发现有 5 个植被指 数能够很好地与物候相吻合, 未来可以利用这几个 指数监测更大尺度的物候信息来分析地中海地区 植被类型分布以及监测植被群落的变化。

近年来, 随着激光雷达遥感技术的发展和激光 雷达获取三维信息的优势, 越来越多的科研单位和 野外台站购置了地基激光雷达用于生态系统结构 提取和生物多样性研究。通过新算法研发, 地基激 光雷达可以更加准确地计算叶面积指数( $\mathrm{Li}$ et al, 2016)和聚集系数(Li et al, 2017)等精细森林结构参 数。除了提取森林结构参数, 许多科研人员尝试使 用地基激光雷达直接对物种进行研究。Azmy等 (2012)利用地基激光雷达扫描仪架设14站对蝙蝠栖 息的100 m长洞穴进行三维扫描, 并构建了洞穴的 三维结构, 通过蝙蝠倒挂在岩壁上的特征成功识别 出蝙蝠并统计了蝙蝠种群数量。地基激光雷达为蝙 蝠栖息地研究提供了新的思路。Yang等(2013)用热 红外相机照片重建了24条棕色大蝙蝠(Eptesicus fuscus) 飞行轨迹, 结合地基激光雷达点云的三维森
林重建, 探究了森林结构性质对蝙蝠受食行为的作 用，结果表明尽管所有蝙蝠的飞行都在回声定位的 控制下避开了树木，但是不同的蝙蝠还是会选择不 同的飞行路线。Shazali等(2017)在马来西亚沙捞越 风洞自然保护区利用地基激光雷达绘制出蝙蝠栖 息洞穴三维结构图, 统计了9种蝙蝠的种群数量, 并根据种群数量大小和栖息模式的关系提出如何 更好地保护蝙蝠。

\section{遥感在生物多样性研究中的局限}

虽然各种遥感手段在生物多样性研究中应用 广泛，能够获取生态系统组成结构和功能信息，但 是仍存在一定的局限性。从遥感技术本身来看, 现 有遥感技术用于生物多样性监测的局限性主要集 中在数据获取与处理方面。

目前, 各遥感平台主要以光学传感器为主, 缺 少反映森林垂直结构的数据源。自从ICESat卫星 2009年10月退役后，距今已有十年没有全球尺度的 森林垂直结构信息数据更新，缺乏服役的卫星限制 了大尺度森林结构参数提取。机载平台受制于高昂 的观测成本和航空管制，只能获取部分区域的激光 雷达和高光谱数据。而且在林型复杂、具有多层结 构的阔叶林, 机载平台获取的激光雷达数据点密度 较低, 无法获取全部的垂直结构信息; 对于茂密的 热带雨林，甚至可能由于地面点不足而导致整个激 光雷达数据无法使用。无人机平台能够获取激光雷 达和高光谱从而直接获取物种信息，反映真实意义 上的生物多样性。但是受限于激光雷达和高光谱传 感器价格, 无人机平台仍以光学相机为主。未来随 着传感器价格下降, 无人机平台在数据获取方面的 局限性将被改善。相对于林冠信息，各遥感平台林 下信息获取不足。除近地面遥感平台中的地基平台 外，其他平台获取的林下信息极其有限，林下信息的 缺失将会限制遥感在地面哺乳动物和昆虫多样性研 究中的应用。

遥感数据的处理难度也给其在生物多样性研 究中的应用带来了一定限制。遥感数据处理的局限 性主要表现为海量数据预处理、空间位置匹配、数 据融合等方面。数据预处理虽然是开展研究的第一 步, 但是对于部分平台来说, 数据量太大直接阻碍 了其应用进度。以地基激光雷达为例，由于观测视 角存在遮挡现象, 监测一块样地需要架设多个站点 


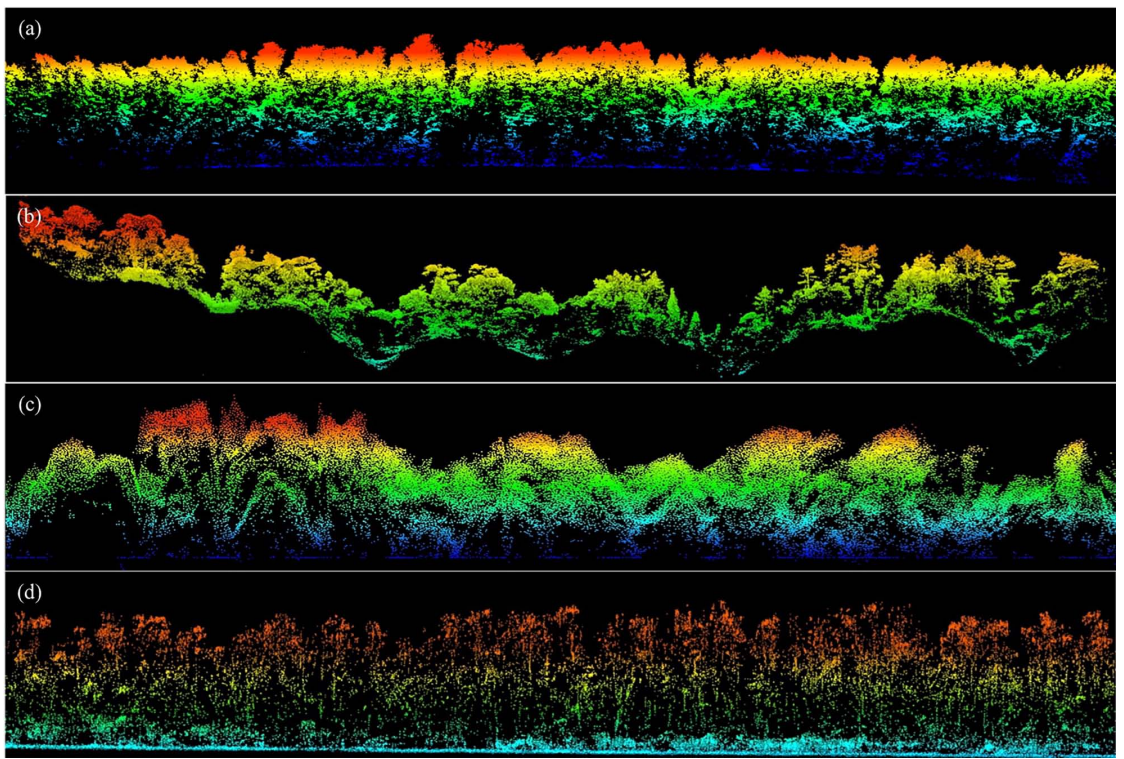

图5 无人机激光雷达获取的不同森林点云剖面图。(a)吉林长白山针阔混交林; (b)浙江古田山常绿阔叶林; (c)云南西双版纳 热带雨林; (d)广东雷州红树林。

Fig. 5 Cloud profile of different forest point clouds obtained by unmanned aerial vehicle lidar. (a) Conifer and broad-leaved mixed forest in Changbai Mountain, Jilin; (b) Evergreen broad-leaved forest in Gutian Mountain, Zhejiang; (c) Tropical rain forest in Xishuangbanna, Yunnan; (d) Mangrove forest in Leizhou, Guangdong.
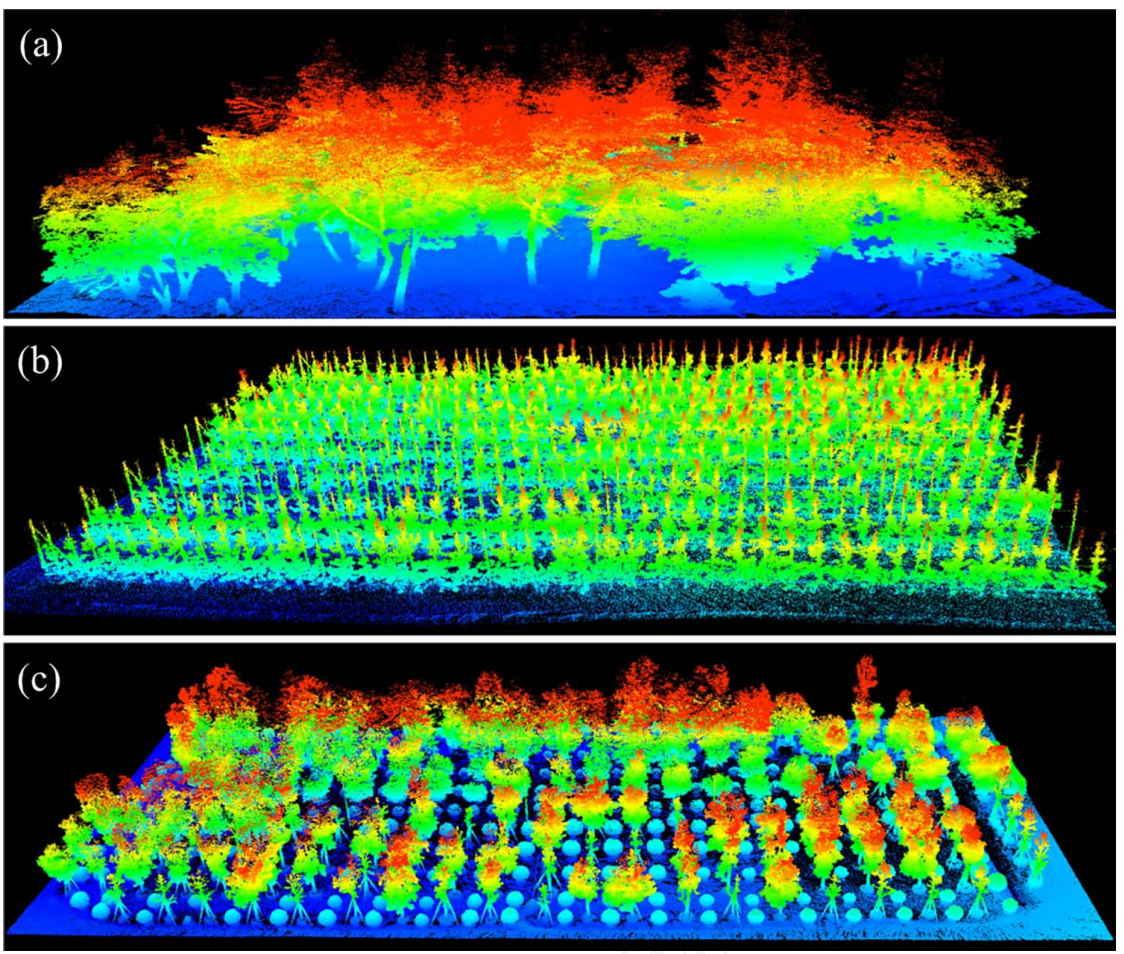

图6 背包激光雷达获取的不同样地的点云数据图。(a)植物园; (b)果园; (c)苗固。

Fig. 6 Plot-level points cloud data obtained by backpack lidar. (a) Botanical Gardens; (b) Orchards; (c) Nurseries.

才能获取完整的数据。但是现在大部分设备对于多 个站点观测数据的拼接无法做到自动化, 拼接工作 主要由人工完成, 耗费大量时间。无人机平台也存
在预处理问题: 目前无人机搭载的多是非量测相机, 难以对相机本身的畸变进行校正, 再加上飞行姿态 的不稳定，影像的畸变较大，同时由于飞行高度 
低、视场小, 获取的影像数量多, 海量数据的配准 以及自动化处理是数据预处理的一大难题。

空间位置匹配的限制一方面来自获取数据设 备本身。如无人机载重较小, 难以集成多种传感器 同平台观测, 无法在硬件上提供不同传感器间的固 连关系, 通常采用多次飞行、搭载不同传感器完成 多源数据的采集, 这类数据需要进行空间位置匹配 才能用于多源数据的融合和分析。而在森林地区激 光雷达数据以及光学数据的自动配准仍是一大难 题。另一方面来自于遥感数据与地面数据的空间位 置不匹配。大多数生物多样性研究在一个相对坐标 系中进行, 并没有采集精确的大地坐标, 因此在与 高分辨率的遥感数据开展分析时总是存在一定的 偏差, 甚至得到错误的结论。

数据融合方面主要是受多源数据之间时空尺 度不匹配、语义不一致等问题限制。高时间分辨率、 高空间分辨率的卫星数据对生物多样性研究具有 重要用途, 然而现有大部分遥感数据存在分辨率较 粗, 高分辨率卫星数据又存在回访时间过长。虽然 能够通过融合高时间分辨率的中低分辨率遥感数 据来解决, 但对于从事生态学研究、遥感基础较弱 的研究人员来说, 却是个棘手的难题。

除了遥感技术本身的局限性外，遥感技术在生 物多样性监测的应用也存在局限性。(1)遥感技术在 不同类群的生物多样性监测研究失衡。目前, 绝大 部分研究均集中在植物多样性, 对于哺乳动物、昆 虫的研究较少; 绝大部分研究关注的是林冠层的多 样性, 而对于林下的多样性研究较少。导致这种失 衡的原因主要取决于数据的可获取性: 林下遥感数 据相对于林冠层的遥感数据更难获取; 动物的生物 多样性数据也比植物多样性难获取。(2)受数据获取 难易程度的限制, 各平台之间的研究数量存在严重 的不平衡。现有研究主要基于卫星遥感平台在较大 尺度开展研究, 而基于近地面遥感和航空遥感的 “样地一景观尺度”的研究较少。遥感数据以影像为主, 未来可以拓展激光雷达、高光谱、热红外等传感器 数据。(3)各平台之间的联合研究较少, 主要是研究 单个尺度的生物多样性问题。虽然现有的平台系统 能够实现不同尺度的生物多样性研究, 但是在同一 地区系统性地利用多个平台开展跨尺度的生物多 样性研究仍非常罕见。然而尺度问题是未来开展标 准的生物多样性监测、管理和评估需要解决的核心
问题之一。(4)生物多样性遥感监测仍缺乏统一的指 标体系用于进行常规和周期性的生物多样性状态 监测及其变化评估。基于光谱变异假说、生产力假 说和生境异质性假说，遥感可以获得直接或间接表 征生物多样性状况的指标。但是，对应不同研究的 空间范围，如何选择适宜的观测平台确立最佳的研 究尺度和观测变量尤为重要。综合考虑爱知目标以 及EBV指标框架体系，在总结遥感技术现阶段可以 稳健获取的各种指标基础上，本文根据不同研究尺 度适宜的观测平台将这些参数从类型、立地条件、 结构和功能 4 个方面进行分类归纳，供以后生物多 样性监测和评估工作遴选应用(表3)。

\section{4 结论与展望}

遥感技术为传统生物多样性研究带来了新的 机遇, 它能够为生物多样性研究提供大尺度、长时 间序列、多类型的观测数据, 是未来生物多样性监 测和评估的重要手段。近地面遥感平台、航空遥感 平台和卫星遥感平台能够为生物多样性研究提供 从样地-景观-区域-洲际-全球尺度的数据, 结合多 元化的传感器能够获取生物多样性研究所需的物 种数量和性状、群落组成以及生态系统功能和结构 等重要信息。

虽然各遥感平台在生物多样性研究与保护中 的应用存在一定的局限性，但是随着新型传感器、 采集平台和分析方法的出现，这些问题将一一解 决。基于SLAM算法的背包激光雷达的出现已经能 够实现林下数据边采集边自动拼接, 而且可以与无 人机数据进行融合从而获取完整的森林信息; 同时 利用背包获取的全景影像数据, 结合物种识别算法, 还能解决传统遥感方法无法获取林下生物多样性 的难题。随着未来电池技术的发展，无人机滞空时 间不断增加将改善现有技术无法长期监测野生动 物的困境。具备长时间滞空特性的无人飞艇或许是 未来动物长期监测的另一种实现方式，通过搭载热 红外传感器和光学传感器长期定位监测观测区中 的哺乳动物。单光子激光雷达的出现和普及, 将解 决航空平台无法获取大范围、高精度激光雷达数据 的困境。在全球尺度，我国即将发射的“林业碳卫 星”获取的激光雷达数据也将弥补现有结构信息的 缺失。未来高空间分辨率、高时间分辨率卫星的增 多，也将为植物多样性和大型哺乳动物的监测提供 
表3 不同空间尺度对应的生物多样性遥感监测关键指标*

Table 3 Biodiversity monitoring indicators based on remote sensing at different spatial scales

\begin{tabular}{|c|c|c|}
\hline $\begin{array}{l}\text { 空间幅度与观测平台 } \\
\text { Scale \& Observation platform }\end{array}$ & $\begin{array}{l}\text { 指标 } \\
\text { Indicators }\end{array}$ & $\begin{array}{l}\text { 参数 } \\
\text { Parameters }\end{array}$ \\
\hline \multirow{4}{*}{$\begin{array}{l}\text { 全球/洲际/国家 } \\
\text { Global/continental/national } \\
\text { 卫星平台 } \\
\text { Satellite borne }\end{array}$} & $\begin{array}{l}\text { 生境类型 } \\
\text { Habitat type }\end{array}$ & $\begin{array}{l}\text { 土地覆盖类型/植被类型/二者结合 } \\
\text { Landcover/vegetation type/both }\end{array}$ \\
\hline & $\begin{array}{l}\text { 立地条件 } \\
\text { Stand condition }\end{array}$ & $\begin{array}{l}\text { 陆面温度 Land surface temperature } \\
\text { 大气降水 Precipitation } \\
\text { 高程、坡度、坡向、坡位 Elevation, slope, aspect, slope position }\end{array}$ \\
\hline & $\begin{array}{l}\text { 生境结构 } \\
\text { Habitat structure }\end{array}$ & $\begin{array}{l}\text { 植被覆盖度 Canopy cover } \\
\text { 植被冠层高度 Canopy height }\end{array}$ \\
\hline & $\begin{array}{l}\text { 生境质量 } \\
\text { Habitat quality }\end{array}$ & $\begin{array}{l}\text { 植被指数 NDVI/EVI/SAVI } \\
\text { 叶面积指数 LAI } \\
\text { 地上生物量 Aboveground biomass } \\
\text { 绿度 Greenness } \\
\text { 光合有效辐射吸收比率 FPAR }\end{array}$ \\
\hline \multirow{4}{*}{$\begin{array}{l}\text { 区域/省际 } \\
\text { Regional/province-scale } \\
\text { 机载平台 } \\
\text { Airborne }\end{array}$} & $\begin{array}{l}\text { 生境类型 } \\
\text { Habitat type }\end{array}$ & $\begin{array}{l}\text { 土地覆盖类型/植被类型/二者结合 } \\
\text { Landcover/vegetation type/both }\end{array}$ \\
\hline & $\begin{array}{l}\text { 立地条件 } \\
\text { Stand condition }\end{array}$ & $\begin{array}{l}\text { 陆面温度 Land surface temperature } \\
\text { 大气降水 Precipitation } \\
\text { 高程、坡度、坡向、坡位 Elevation, slope, aspect, slope position }\end{array}$ \\
\hline & $\begin{array}{l}\text { 生境结构 } \\
\text { Habitat structure }\end{array}$ & $\begin{array}{l}\text { 植被覆盖度 Canopy cover } \\
\text { 植被冠层高度 Canopy height }\end{array}$ \\
\hline & $\begin{array}{l}\text { 生境质量 } \\
\text { Habitat quality }\end{array}$ & $\begin{array}{l}\text { 植被指数 NDVI/EVI/SAVI } \\
\text { 叶面积指数 LAI } \\
\text { 地上生物量 Aboveground biomass } \\
\text { 绿度 Greenness } \\
\text { 光合有效辐射吸收比率 FPAR }\end{array}$ \\
\hline \multirow{4}{*}{$\begin{array}{l}\text { 景观 } \\
\text { Landscape } \\
\text { 无人机平台 } \\
\text { UAV borne }\end{array}$} & $\begin{array}{l}\text { 生境类型 } \\
\text { Habitat type }\end{array}$ & $\begin{array}{l}\text { 植被类型 Vegetation type } \\
\text { 景观多样性指数 Landscape diversity index }\end{array}$ \\
\hline & $\begin{array}{l}\text { 立地条件 } \\
\text { Stand condition }\end{array}$ & $\begin{array}{l}\text { 陆面温度 Land surface temperature } \\
\text { 大气降水 Precipitation } \\
\text { 高程、坡度、坡向、坡位 Elevation, slope, aspect, slope position } \\
\text { 土壤含水量 Soil water content }\end{array}$ \\
\hline & $\begin{array}{l}\text { 生境结构 } \\
\text { Habitat structure }\end{array}$ & $\begin{array}{l}\text { 植被覆盖度 Canopy cover } \\
\text { 植被冠层高度 Canopy height } \\
\text { 植被密度 Vegetation density } \\
\text { 斑块大小、形状、丰富度 Size, shape and richness of patches }\end{array}$ \\
\hline & $\begin{array}{l}\text { 生境质量 } \\
\text { Habitat quality }\end{array}$ & $\begin{array}{l}\text { 植被指数 NDVI/EVI/SAVI } \\
\text { 叶面积指数 LAI } \\
\text { 地上生物量 Aboveground biomass } \\
\text { 绿度 Greenness } \\
\text { 光合有效辐射吸收比率 FPAR } \\
\text { 景观聚集度指数 Landscape aggregation metrics } \\
\text { 景观连通性指数 Landscape connectivity metrics } \\
\text { 景观破碎化程度 Landscape fragmentation index }\end{array}$ \\
\hline \multirow{3}{*}{$\begin{array}{l}\text { 局地/样地 } \\
\text { Local/plot } \\
\text { 地基移动/固定平台 } \\
\text { Terrestrial or mobile platform }\end{array}$} & $\begin{array}{l}\text { 生境类型 } \\
\text { Habitat type }\end{array}$ & $\begin{array}{l}\text { 植被类型 Vegetation type } \\
\text { 物种多样性指数 Biodiversity index }\end{array}$ \\
\hline & $\begin{array}{l}\text { 立地条件 } \\
\text { Stand condition }\end{array}$ & $\begin{array}{l}\text { 土壤含水量 Soil water content } \\
\text { 高程、坡度、坡向、坡位 Elevation, slope, aspect, slope position }\end{array}$ \\
\hline & $\begin{array}{l}\text { 生境结构 } \\
\text { Habitat structure }\end{array}$ & $\begin{array}{l}\text { 植被覆盖度 Canopy cover } \\
\text { 冠层高度 Canopy height } \\
\text { 植被密度 Vegetation density } \\
\text { 单木树高 Individual tree height } \\
\text { 枝下高 Crown base height } \\
\text { 植被冠层高度剖面 Vegetation height profile } \\
\text { 冠幅 Crown size }\end{array}$ \\
\hline
\end{tabular}


表3(续) Table 3 (continued)

\begin{tabular}{lll}
\hline $\begin{array}{l}\text { 空间幅度与观测平台 } \\
\text { Scale \& Observation platform }\end{array}$ & $\begin{array}{l}\text { 参数 } \\
\text { Indicators }\end{array}$ & Parameters \\
\hline & 生境质量 & 植被指数 NDVI/EVI/SAVI \\
& Habitat quality & 叶面积指数 LAI \\
& & 地上生物量 Aboveground biomass \\
& & 绿度 Greenness \\
& 光合有效辐射吸收比率FPAR \\
& 景观聚集度指数 Landscape aggregation metrics \\
& 景观连通性指数 Landscape connectivity metrics
\end{tabular}

*指标参考以下文献: Turner et al, 2003; Duro et al, 2007; Dassot et al, 2011; Goetz et al, 2014

重要的数据源。此外, 新兴遥感技术也能从其他维 度丰富生物多样性的信息。例如, 叶绿素苂光遥感 能够反映植被的光合作用, 可以从生产力假说的角 度服务于生物多样性监测。

随着遥感平台和生物多样性监测技术发展, 遥 感和生物多样性数据将不断地丰富和完善, 传统基 于小样本的分析方法将难以适用, 深度学习等大数 据分析方法的出现也将为未来生物多样性监测和 研究提供新的思路。在大数据时代下, 需要生态学 家和遥感学家共享各自领域的数据, 开展深入的合 作，整合各自优势更好地服务于生物多样性保护和 评估。

致谢：感谢中国生物多样性监测与研究网络的 支持。

\section{参考文献}

Adame K, Pardo MA, Salvadeo C, Beier E, ElorriagaVerplancken FR (2017) Detectability and categorization of California sea lions using an unmanned aerial vehicle. Marine Mammal Science, 33, 913-925.

Asner G, Martin R, Knapp D, Tupayachi R, Anderson C, Sinca F, Vaughn N, Llactayo W (2017) Airborne laser-guided imaging spectroscopy to map forest trait diversity and guide conservation. Science, 355, 385-389.

Asner GP, Martin RE (2008) Spectral and chemical analysis of tropical forests: Scaling from leaf to canopy levels. Remote Sensing of Environment, 112, 3958-3970.

Austin GE, Thomas CJ, Houston DC, Thompson DB (1996) Predicting the spatial distribution of buzzard Buteo buteo nesting areas using a geographical information system and remote sensing. Journal of Applied Ecology, 33, 1541-1550.

Azmy SN, Sah SAM, Shafie NJ, Ariffin A, Majid Z, Ismail MNA, Shamsir MS (2012) Counting in the dark: Non-intrusive laser scanning for population counting and identifying roosting bats. Scientific Reports, 2, 524.
Brisson-Curadeau E, Bird D, Burke C, Fifield DA, Pace P, Sherley RB, Elliott KH (2017) Seabird species vary in behavioural response to drone census. Scientific Reports, 7, 17884.

Carleer A, Wolff E (2004) Exploitation of very high resolution satellite data for tree species identification. Photogrammetric Engineering \& Remote Sensing, 70, 135-140.

Carlson KM, Asner GP, Hughes RF, Ostertag R, Martin RE (2007) Hyperspectral remote sensing of canopy biodiversity in Hawaiian lowland rainforests. Ecosystems, 10, 536-549.

Ceballos G, Ehrlich PR, Barnosky AD, García A, Pringle RM, Palmer TM (2015) Accelerated modern human-induced species losses: Entering the sixth mass extinction. Science Advances, 1, e1400253.

Clawges R, Vierling K, Vierling L, Rowell E (2008) The use of airborne lidar to assess avian species diversity, density, and occurrence in a pine/aspen forest. Remote Sensing of Environment, 112, 2064-2073.

Clerici N, Weissteiner CJ, Gerard F (2012) Exploring the use of MODIS NDVI-based phenology indicators for classifying forest general habitat categories. Remote Sensing, 4, 1781-1803.

Coops NC, Tompaski P, Nijland W, Rickbeil GJ, Nielsen SE, Bater CW, Stadt JJ (2016) A forest structure habitat index based on airborne laser scanning data. Ecological Indicators, 67, 346-357.

Coops NC, Wulder MA, Duro DC, Han T, Berry S (2008) The development of a Canadian dynamic habitat index using multi-temporal satellite estimates of canopy light absorbance. Ecological Indicators, 8, 754-766.

Dassot M, Constant T, Fournier M (2011) The use of terrestrial LiDAR technology in forest science: Application fields, benefits and challenges. Annals of Forest Science, 68, 959-974.

Davies AB, Asner GP (2014) Advances in animal ecology from 3D-LiDAR ecosystem mapping. Trends in Ecology \& Evolution, 29, 681-691.

Duffy JP, Pettorelli N (2012) Exploring the relationship between NDVI and African elephant population density in protected areas. African Journal of Ecology, 50, 455-463.

Duro DC, Coops NC, Wulder MA, Han T (2007) Development 
of a large area biodiversity monitoring system driven by remote sensing. Progress in Physical Geography: Earth and Environment, 31, 235-260.

Evans LJ, Jones TH, Pang K, Evans MN, Saimin S, Goossens B (2015) Use of drone technology as a tool for behavioral research: A case study of crocodilian nesting. Herpetological Conservation and Biology, 10, 90-98.

Farashi A, Parvian N, Najafabadi MS (2016) Land use and land cover change in protected areas: Using remote sensing to survey suitable habitats of brown bear Ursus arctos. Polish Journal of Ecology, 64, 420-430.

Franklin KA, Lyons K, Nagler PL, Lampkin D, Glenn EP, Molina-Freaner F, Markow T, Huete AR (2006) Buffelgrass (Pennisetum ciliare) land conversion and productivity in the plains of Sonora, Mexico. Biological Conservation, 127, 62-71.

Fuentes S, Palmer AR, Taylor D, Zeppel M, Whitley R, Eamus $\mathrm{D}$ (2008) An automated procedure for estimating the leaf area index (LAI) of woodland ecosystems using digital imagery, MATLAB programming and its application to an examination of the relationship between remotely sensed and field measurements of LAI. Functional Plant Biology, 35, 1070-1079.

Ganguly S, Friedl MA, Tan B, Zhang X, Verma M (2010) Land surface phenology from MODIS: Characterization of the Collection 5 global land cover dynamics product. Remote Sensing of Environment, 114, 1805-1816.

Getzin S, Wiegand K, Schoening I (2012) Assessing biodiversity in forests using very high-resolution images and unmanned aerial vehicles. Methods in Ecology and Evolution, 3, 397-404.

Gillespie TW, Foody GM, Rocchini D, Giorgi AP, Saatchi S (2008) Measuring and modelling biodiversity from space. Progress in Physical Geography, 32, 203-221.

Godinho S, Gil A, Guiomar N, Neves N, Pinto-Correia T (2016) A remote sensing-based approach to estimating montado canopy density using the FCD model: A contribution to identifying HNV farmlands in southern Portugal. Agroforestry Systems, 90, 23-34.

Goetz S, Sun M, Zolkos S, Hansen A, Dubayah R (2014) The relative importance of climate and vegetation properties on patterns of North American breeding bird species richness. Environmental Research Letters, 9, 034013.

Goetz SJ, Steinberg D, Betts MG, Holmes RT, Doran PJ, Dubayah R, Hofton M (2010) Lidar remote sensing variables predict breeding habitat of a Neotropical migrant bird. Ecology, 91, 1569-1576.

Gould W (2000) Remote sensing of vegetation, plant species richness, and regional biodiversity hotspots. Ecological Applications, 10, 1861-1870.

Guo QH, Liu J, Li YM, Zhai QP, Wang YC, Wu FF, Hu TY, Wan HW, Liu HM, Shen WM (2016a) A near-surface remote sensing platform for biodiversity monitoring: Perspectives and prospects. Biodiversity Science, 24, 1249-1266. (in Chinese with English abstract) [郭庆华, 刘瑾, 李玉美, 翟 秋萍, 王永财, 吴芳芳, 胡天宇, 万华伟, 刘慧明, 申文 明 (2016a) 生物多样性近地面遥感监测: 应用现状与前 景展望. 生物多样性, 24, 1249-1266.]

Guo QH, Wu FF, Hu TY, Chen LM, Liu J, Zhao XQ, Gao S, Pang SX (2016b) Perspectives and prospects of unmanned aerial vehicle in remote sensing monitoring of biodiversity. Biodiversity Science, 24, 1267-1278. (in Chinese with English abstract) [郭庆华, 吴芳芳, 胡天宇, 陈琳海, 刘瑾, 赵晓倩, 高上, 庞树金金 (2016b) 无人机在生物多样性遥 感监测中的应用现状与展望. 生物多样性, 24, 1267-1278.]

Handcock RN, Swain DL, Bishop-Hurley GJ, Patison KP, Wark T, Valencia P, Corke P, O’Neill CJ (2009) Monitoring animal behaviour and environmental interactions using wireless sensor networks, GPS collars and satellite remote sensing. Sensors, 9, 3586-3603.

Hernández-Stefanoni JL, Dupuy JM (2007) Mapping species density of trees, shrubs and vines in a tropical forest, using field measurements, satellite multiespectral imagery and spatial interpolation. Biodiversity and Conservation, 16, 3817-3833.

Hodgson A, Kelly N, Peel D (2013) Unmanned aerial vehicles (UAVs) for surveying marine fauna: A Dugong case study. PLoS ONE, 8, e79556.

Hu JB, Zhang J (2018) Unmanned aerial vehicle remote sensing in ecology: Advances and prospects. Acta Ecologica Sinica, 38, 20-30. (in Chinese with English abstract) [胡健 波, 张健 (2018) 无人机遥感在生态学中的应用进展. 生 态学报, 38, 20-30.]

Hu T, Su Y, Xue B, Liu J, Zhao X, Fang J, Guo Q (2016) Mapping global forest aboveground biomass with spaceborne LiDAR, optical imagery, and forest inventory data. Remote Sensing, 8, 565.

Hutchinson J, Jacquin A, Hutchinson S, Verbesselt J (2015) Monitoring vegetation change and dynamics on US Army training lands using satellite image time series analysis. Journal of Environmental Management, 150, 355-366.

Jordan YC, Ghulam A, Herrmann RB (2012) Floodplain ecosystem response to climate variability and land-cover and land-use change in Lower Missouri River basin. Landscape Ecology, 27, 843-857.

Kalacska M, Arroyo-Mora JP, de Gea J, Snirer E, Herzog C, Moore TR (2013) Videographic analysis of Eriophorum vaginatum spatial coverage in an ombotrophic bog. Remote Sensing, 5, 6501-6512.

Kerr JT, Ostrovsky M (2003) From space to species: Ecological applications for remote sensing. Trends in Ecology \& Evolution, 18, 299-305.

Kerr JT, Southwood T, Cihlar J (2001) Remotely sensed habitat diversity predicts butterfly species richness and community similarity in Canada. Proceedings of the National Academy 
of Sciences, USA, 98, 11365-11370.

Kiszka JJ, Mourier J, Gastrich K, Heithaus MR (2016) Using unmanned aerial vehicles (UAVs) to investigate shark and ray densities in a shallow coral lagoon. Marine Ecology Progress Series, 560, 237-242.

Knudby A, LeDrew E, Brenning A (2010) Predictive mapping of reef fish species richness, diversity and biomass in Zanzibar using IKONOS imagery and machine-learning techniques. Remote Sensing of Environment, 114, 1230-1241.

Krause DJ, Hinke JT, Perryman WL, Goebel ME, LeRoi DJ (2017) An accurate and adaptable photogrammetric approach for estimating the mass and body condition of pinnipeds using an unmanned aerial system. PLoS ONE, 12, e0187465.

Lausch A, Bannehr L, Beckmann M, Boehm C, Feilhauer H, Hacker JM, Heurichr M, Jung A, Klenke R, Neumann C, Pause M, Rocchini D, Schaepman ME, Schmidtlein S, Schulz K, Selsam P, Settele J, Skidmore AK, Cord AF (2016) Linking Earth Observation and taxonomic, structural and functional biodiversity: Local to ecosystem perspectives. Ecological Indicators, 70, 317-339.

Leyequien E, Verrelst J, Slot M, Schaepman-Strub G, Heitkönig IM, Skidmore A (2007) Capturing the fugitive: Applying remote sensing to terrestrial animal distribution and diversity. International Journal of Applied Earth Observation and Geoinformation, 9, 1-20.

Li S, Wang DJ, Xiao ZS, Li XH, Wang TM, Feng LM, Wang $\mathrm{Y}$ (2014) Camera-trapping in wildlife research and conservation in China: Review and outlook. Biodiversity Science, 22, 685-695. (in Chinese with English abstract) [李晟, 王 大军, 肖治术, 李欣海, 王天明, 冯利民, 王云 (2014) 红外相机技术在我国野生动物研究与保护中的应用与前 景. 生物多样性, 22, 685-695.]

Li SQ, Wang GH, Shi ZP, Li XK, Xiao ZS, Zhou QH (2016) Infrared camera traps for monitoring mammal and bird diversity and activity pattern in limestone habitats. Acta Theriologica Sinica, 36, 272-281. (in Chinese with English abstract) [李生强, 汪国海, 施泽攀, 李先琨, 肖治术, 周岐 海 (2016) 红外相机技术监测喀斯特生境兽类和鸟类多 样性及活动节律. 兽类学报, 36, 272-281.]

Li Y, Guo Q, Su Y, Tao S, Zhao K, Xu G (2017) Retrieving the gap fraction, element clumping index, and leaf area index of individual trees using single-scan data from a terrestrial laser scanner. ISPRS Journal of Photogrammetry and Remote Sensing, 130, 308-316.

Li Y, Guo Q, Tao S, Zheng G, Zhao K, Xue B, Su Y (2016) Derivation, validation, and sensitivity analysis of terrestrial laser scanning-based leaf area index. Canadian Journal of Remote Sensing, 42, 719-729.

Loh J, Green RE, Ricketts T, Lamoreux J, Jenkins M, Kapos V, Randers J (2005) The living planet index: Using species population time series to track trends in biodiversity. Philosophical Transactions of the Royal Society B: Biological
Sciences, 360, 289-295.

Löffler E, Margules C (1980) Wombats detected from space. Remote Sensing of Environment, 9, 47-56.

Lucas KL, Raber GT, Carter GA (2010) Estimating vascular plant species richness on Horn Island, Mississippi using small-footprint airborne LiDAR. Journal of Applied Remote Sensing, 4, 043545.

Luschi P, Hays G, Del Seppia C, Marsh R, Papi F (1998) The navigational feats of green sea turtles migrating from Ascension Island investigated by satellite telemetry. Proceedings of the Royal Society of London B: Biological Sciences, 265, 2279-2284.

Ma KP (1993) On the concept of biodiversity. Chinese Biodiversity, 1, 20-22. (in Chinese) [马克平 (1993) 试论生物多 样性的概念. 生物多样性, 1, 20-22.]

Martin M, Newman S, Aber J, Congalton R (1998) Determining forest species composition using high spectral resolution remote sensing data. Remote Sensing of Environment, 65, 249-254.

Mishra NB, Chaudhuri G (2015) Spatio-temporal analysis of trends in seasonal vegetation productivity across Uttarakhand, Indian Himalayas, 2000-2014. Applied Geography, 56, 29-41.

Mulero-Pazmany M, Jenni-Eiermann S, Strebel N, Sattler T, Jose Negro J, Tablado Z (2017) Unmanned aircraft systems as a new source of disturbance for wildlife: A systematic review. PLoS ONE, 12, e0178448.

Mulero-Pazmany M, Stolper R, van Essen LD, Negro JJ, Sassen $\mathrm{T}$ (2014) Remotely piloted aircraft systems as a rhinoceros anti-poaching tool in Africa. PLoS ONE, 9, e83873.

Müller J, Brandl R (2009) Assessing biodiversity by remote sensing in mountainous terrain: The potential of LiDAR to predict forest beetle assemblages. Journal of Applied Ecology, 46, 897-905.

Myers N, Mittermeier RA, Mittermeier CG, da Fonseca GAB, Kent J (2000) Biodiversity hotspots for conservation priorities. Nature, 403, 853-858.

Nicholson E, Mace GM, Armsworth PR, Atkinson G, Buckle S, Clements T, Ewers RM, Fa JE, Gardner TA, Gibbons J, Grenyer R, Metcalfe R, Mourato S, Muuls M, Osborn D, Reuman DC, Watson C, Milner-Gulland EJ (2009) Priority research areas for ecosystem services in a changing world. Journal of Applied Ecology, 46, 1139-1144

Ojoyi MM, Mutanga O, Odindi J, Kahinda JMM, Abdel-Rahman EM (2017) Implications of land use transitions on soil nitrogen in dynamic landscapes in Tanzania. Land Use Policy, 64, 95-100.

Paganini M, Leidner AK, Geller G, Turner W, Wegmann M (2016) The role of space agencies in remotely sensed essential biodiversity variables. Remote Sensing in Ecology and Conservation, 2, 132-140.

Pereira HM, Ferrier S, Walters M, Geller GN, Jongman R, Scholes RJ, Bruford MW, Brummitt N, Butchart S, Cardoso 
A (2013) Essential biodiversity variables. Science, 339, 277-278.

Pettorelli N, Safi K, Turner W (2014) Satellite remote sensing, biodiversity research and conservation of the future. Philosophical Transactions of the Royal Society of London, 369, 20130190.

Purkis SJ, Graham N, Riegl B (2008) Predictability of reef fish diversity and abundance using remote sensing data in Diego Garcia (Chagos Archipelago). Coral Reefs, 27, 167-178.

Ramankutty N, Foley JA (1999) Estimating historical changes in land cover: North American croplands from 1850 to 1992. Global Ecology and Biogeography, 8, 381-396.

Rocchini D, Ricotta C, Chiarucci A (2007) Using satellite imagery to assess plant species richness: The role of multispectral systems. Applied Vegetation Science, 10, 325-331.

Roughgarden J, Running SW, Matson PA (1991) What does remote sensing do for ecology? Ecology, 72, 1918-1922.

Saatchi S, Agosti D, Alger K, Delabie J, Musinsky J (2001) Examining fragmentation and loss of primary forest in the southern Bahian Atlantic forest of Brazil with radar imagery. Conservation Biology, 15, 867-875.

Saatchi S, Buermann W, Ter Steege H, Mori S, Smith TB (2008) Modeling distribution of Amazonian tree species and diversity using remote sensing measurements. Remote Sensing of Environment, 112, 2000-2017.

Sasaki T, Imanishi J, Ioki K, Morimoto Y, Kitada K (2012) Object-based classification of land cover and tree species by integrating airborne LiDAR and high spatial resolution imagery data. Landscape and Ecological Engineering, 8, 157-171.

Sarda-Palomera F, Bota G, Vinolo C, Pallares O, Sazatornil V, Brotons L, Gomariz S, Sarda F (2012) Fine-scale bird monitoring from light unmanned aircraft systems. Ibis, 154, 177-183.

Saveraid EH, Debinski DM, Kindscher K, Jakubauskas ME (2001) A comparison of satellite data and landscape variables in predicting bird species occurrences in the Greater Yellowstone Ecosystem, USA. Landscape Ecology, 16, 71-83.

Schofield G, Katselidis KA, Lilley MKS, Reina RD, Hays GC (2017) Detecting elusive aspects of wildlife ecology using drones: New insights on the mating dynamics and operational sex ratios of sea turtles. Functional Ecology, 31, 2310-2319.

Sequeira AM, Mellin C, Fordham DA, Meekan MG, Bradshaw CJ (2014) Predicting current and future global distributions of whale sharks. Global Change Biology, 20, 778-789.

Shazali N, Chew TH, Shamsir MS, Tingga RCT, Mohd-Ridwan AR, Khan FAA (2017) Assessing bat roosts using the LiDAR system at Wind Cave Nature Reserve in Sarawak, Malaysian Borneo. Acta Chiropterologica, 19, 199-210.

Shirley S, Yang Z, Hutchinson R, Alexander J, McGarigal K,
Betts M (2013) Species distribution modelling for the people: Unclassified landsat TM imagery predicts bird occurrence at fine resolutions. Diversity and Distributions, 19, 855-866.

Simonson WD, Allen HD, Coomes DA (2012) Use of an airborne lidar system to model plant species composition and diversity of Mediterranean oak forests. Conservation Biology, 26, 840-850.

Skidmore AK, Pettorelli N (2015) Agree on biodiversity metrics to track from space: Ecologists and space agencies must forge a global monitoring strategy. Nature, 523, 403-406.

Skole D, Tucker C (1993) Tropical deforestation and habitat fragmentation in the Amazon: Satellite data from 1978 to 1988. Science, 260, 1905-1910.

Su YJ, Guo QH, Xue BL, Hu TY, Alvarez O, Tao SL, Fang JY (2016a) Spatial distribution of forest aboveground biomass in China: Estimation through combination of spaceborne Lidar, optical imagery, and forest inventory data. Remote Sensing of Environment, 173, 187-199.

Su YJ, Guo Q, Fry DL, Collins BM, Kelly M, Flanagan JP, Battles JJ (2016b) A vegetation mapping strategy for conifer forests by combining airborne LiDAR data and aerial imagery. Canadian Journal of Remote Sensing, 42, 1-15.

Su YJ, Ma Q, Guo Q (2017) Fine-resolution forest tree height estimation across the Sierra Nevada through the integration of spaceborne LiDAR, airborne LiDAR, and optical imagery. International Journal of Digital Earth, 10, 307-323.

Szantoi Z, Smith SE, Strona G, Koh LP, Wich SA (2017) Mapping orangutan habitat and agricultural areas using Landsat OLI imagery augmented with unmanned aircraft system aerial photography. International Journal of Remote Sensing, 38, 2231-2245.

Tempel DJ, Gutiérrez RJ, Battles JJ, Fry DL, Su Y, Guo Q, Reetz MJ, Whitmore SA, Jones GM, Collins BM, Stephens SL, Kelly M, Berigan WJ, Peery MZ (2015) Evaluating short- and long-term impacts of fuels treatments and simulated wildfire on an old-forest species. Ecosphere, 6, 1-18.

Tuanmu MN, Jetz W (2015) A global, remote sensing-based characterization of terrestrial habitat heterogeneity for biodiversity and ecosystem modelling. Global Ecology and Biogeography, 24, 1329-1339.

Turner W, Spector S, Gardiner N, Fladeland M, Sterling E, Steininger M (2003) Remote sensing for biodiversity science and conservation. Trends in Ecology \& Evolution, 18, 306-314.

Vermeulen C, Lejeune P, Lisein J, Sawadogo P, Bouche P (2013) Unmanned aerial survey of elephants. PLoS ONE, 8, e54700.

Vihervaara P, Mononen L, Auvinen A-P, Virkkala R, Lü Y, Pippuri I, Packalen P, Valbuena R, Valkama J (2015) How to integrate remotely sensed data and biodiversity for ecosystem assessments at landscape scale. Landscape Ecology, 30, 501-516. 
Wang Y, Moskovits DK (2001) Tracking fragmentation of natural communities and changes in land cover: Applications of Landsat data for conservation in an urban landscape (Chicago Wilderness). Conservation Biology, 15, 835-843.

Weil G, Lensky IM, Levin N (2017) Using ground observations of a digital camera in the VIS-NIR range for quantifying the phenology of Mediterranean woody species. International Journal of Applied Earth Observation and Geoinformation, 62, 88-101.

Wilson BT, Lister AJ, Riemann RI (2012) A nearest-neighbor imputation approach to mapping tree species over large areas using forest inventory plots and moderate resolution raster data. Forest Ecology and Management, 271, 182-198.

Yang X, Schaaf C, Strahler A, Kunz T, Fuller N, Betke M, Wu Z, Wang Z, Theriault D, Culvenor D, Jupp D, Newnham G, Lovell J (2013) Study of bat flight behavior by combining thermal image analysis with a LiDAR forest reconstruction. Canadian Journal of Remote Sensing, 39, S112-S125.

Zhang J, Hu J, Lian J, Fan Z, Ouyang X, Ye W (2016) Seeing the forest from drones: Testing the potential of lightweight drones as a tool for long-term forest monitoring. Biological Conservation, 198, 60-69.

Zhao F, Sweitzer RA, Guo Q, Kelly M (2012) Characterizing habitats associated with fisher den structures in the Southern
Sierra Nevada, California using discrete return lidar. Forest Ecology and Management, 280, 112-119.

Zhao Y, Feng D, Yu L, Wang X, Chen Y, Bai Y, Hernández H J, Galleguillos M, Estades C, Biging GS (2016) Detailed dynamic land cover mapping of Chile: Accuracy improvement by integrating multi-temporal data. Remote Sensing of Environment, 183, 170-185.

Zhao Y, Zeng Y, Zheng Z, Dong W, Zhao D, Wu B, Zhao Q (2018) Forest species diversity mapping using airborne LiDAR and hyperspectral data in a subtropical forest in China. Remote Sensing of Environment, 213, 104-114.

Zhou L, Tian Y, Myneni RB, Ciais P, Saatchi S, Liu YY, Piao S, Chen H, Vermote EF, Song C (2014) Widespread decline of Congo rainforest greenness in the past decade. Nature, 509, 86-90.

Zhu C, Fang Y, Zhou KX, Mu SJ, Jiang JL (2015) IUCN red list of ecosystems: a new tool for biodiversity conservation. Acta Ecologica Sinica, 35, 2826-2836. (in Chinese with English abstract) [朱超, 方颖, 周可新, 穆少杰, 蒋金亮 (2015) IUCN生态系统红色名录一一一种新的生物多样性 保护工具. 生态学报, 35, 2826-2836. ]

(责任编委：唐志尧 责任编辑：时意专) 